\title{
Correlations in Perturbed Dual-Unitary Circuits: Efficient Path-Integral Formula
}

\author{
Pavel Kos@, Bruno Bertini®, and Tomaž Prosen $\odot$ \\ Department of Physics, Faculty of Mathematics and Physics, University of Ljubljana, \\ Jadranska 19, SI-1000 Ljubljana, Slovenia
}

(Received 17 June 2020; revised 26 October 2020; accepted 15 December 2020; published 3 February 2021)

\begin{abstract}
Interacting many-body systems with explicitly accessible spatiotemporal correlation functions are extremely rare, especially in the absence of Bethe-ansatz or Yang-Baxter integrability. Recently, we identified a remarkable class of such systems and termed them dual-unitary quantum circuits. These are brickwork-type local quantum circuits whose dynamics are unitary in both time and space. The spatiotemporal correlation functions of these systems turn out to be nontrivial only at the edge of the causal light cone and can be computed in terms of one-dimensional transfer matrices. Dual unitarity, however, requires fine-tuning, and the degree of generality of the observed dynamical features remains unclear. Here, we address this question by studying perturbed dual-unitary quantum circuits. Considering arbitrary perturbations of the local gates, we prove that for a particular class of unperturbed elementary dual-unitary gates the correlation functions are still expressed in terms of one-dimensional transfer matrices. These matrices, however, are now contracted over generic paths connecting the origin to a fixed end point inside the causal light cone. The correlation function is given as a sum over all such paths. Our statement is rigorous in the "dilute limit," where only a small fraction of the gates is perturbed, and in the presence of random longitudinal fields, but we provide theoretical arguments and stringent numerical checks supporting its validity even in the clean case (no randomness) and when all gates are perturbed. As a by-product of our analysis, in the case of random longitudinal fields-which turns out to be equivalent to certain classical Markov chains-we find four types of non-dual-unitary (and nonintegrable) interacting many-body systems where the correlation functions are exactly solvable and given-without approximations-by the path-sum formula.
\end{abstract}

DOI: 10.1103/PhysRevX.11.011022

Subject Areas: Quantum Physics, Statistical Physics

\section{INTRODUCTION}

Understanding the dynamics of extended quantum many-body systems with local interactions is the core problem of nonequilibrium statistical mechanics, with a wide range of applications ranging from condensed matter physics to high-energy theory and quantum gravity. In particular, the set of two-point spatiotemporal correlation functions of local observables can be considered as the prime quantifier of the dynamics. For example, they can be used in the framework of linear response theory $[1,2]$ to express coefficients, such as conductivities and kinematic viscosities, that describe macroscopic transport properties.

A major obstacle is that computing dynamical correlation functions in interacting systems is notoriously hard. This is true for numerical simulations of correlations in real time, which are typically exponentially hard

Published by the American Physical Society under the terms of the Creative Commons Attribution 4.0 International license. Further distribution of this work must maintain attribution to the author(s) and the published article's title, journal citation, and DOI. (in physical simulation time) [3,4] (see also Ref. [5]), and even more so for analytical computations. Even in Bethe-ansatz integrable systems, the task turns out to be daunting: While recent breakthroughs allow for the calculation of dynamical correlations in the late-time, hydrodynamic, regime [6-8], the computation of two-point correlations at arbitrary intermediate times remains out of reach. This fact leaves us with noninteracting, quasi-free, or Gaussian theories as the only general class of systems where dynamical correlations can be analytically computed, in general. Dynamical correlations in coupled (interacting) theories are then usually formulated in terms of (Keldysh) diagrammatic many-body perturbation theory [1]. In these approaches, correlations are written as power series in the coupling constant around the underlaying free theory. Such perturbative series, however, generically have a vanishing radius of convergence-especially in the thermodynamic limit — and their relevance for determining the actual physical behavior of correlation functions in extended systems is questionable. One typically finds an even qualitative change in the behavior of correlations and transport coefficients at finite temperatures when going from free to strongly coupled theories. For example, while 
free theories usually behave as ballistic conductors, strongly coupled ones are expected to display diffusive transport, which is, thus, a nonperturbative effect. In fact, establishing the microscopic origin of diffusion remains one of the main challenges of statistical physics $[9,10]$.

Very recently, we proposed a new class of locally interacting many-body systems, which allows for an exact computation of spatiotemporal correlation functions of local observables [11] and of some other indicators of quantum chaos and scrambling of quantum information [12-21]. These systems are special brickwork-type quantum circuits (see, e.g., Refs. [22-24]) called "dual-unitary" quantum circuits and are characterized by unitary evolution, not only in the direction of time propagation, but also in the orthogonal direction of space propagation. Owing to this property, dual-unitary systems allow for a conceptual exchange of space and time axes and, in a vague sense, are discrete-space-time analogs of conformal field theories. In particular, the correlations in these systems can propagate only at the maximal speed and along one-dimensional straight paths in the space-time. One may think of these models as being "statistically exactly solvable," and, yet, their dynamics is generically ergodic [11] and quantum chaotic, from the point of view of both spectral statistics [12] and dynamical complexity $[13,15]$. This situation is similar to that in classical dynamical systems [25], where correlation functions of certain strongly chaotic singleparticle models, such as, e.g., Arnold cat maps or Baker maps, are exactly computable [26,27] despite their deterministic trajectories being unpredictable and "incomputable" in the algorithmic complexity sense. Similarly, for generic quantum chaotic dual-unitary dynamics, the Heisenberg evolution of local operators is exponentially hard to simulate (classically) $[13,15]$ even though the twopoint functions at infinite temperature are computable exactly (i.e., with vanishing algorithmic complexity).

Dual unitarity, however, requires fine-tuning of coupling parameters in the elementary two-body gates defining the systems, and it is, thus, clearly not stable under generic perturbations. Nonetheless, one might wonder whether the properties of ergodicity and quantum chaos of dual-unitary circuits are structurally stable in a similar way as in classical ergodic theory. There, one can prove that linear automorphisms on the tori (Arnold cat maps) are topologically (structurally) stable under perturbations [28]. A quantitative measure of such stability may be a uniform continuity of two-point time correlation functions with respect to the perturbation parameter.

While one may imagine integrable and free extended quantum many-body systems as isolated points or lowdimensional submanifolds (clearly structurally unstable in the above sense) in some high-dimensional manifold of all appropriate (say, translationally invariant, locally interacting, etc.) systems, the chaotic and ergodic models may represent a finite domain with positive measure. As a matter of fact, the prevailing opinion of the scientific community is that almost all models are ergodic, depending on the precise definition of the ergodic class.

In this paper, we make the first step in addressing the question of structural stability by studying correlation functions in perturbed dual-unitary circuits. We consider generic perturbations on two different types of dual-unitary circuits: (i) noisy circuits on qubits - there are random local longitudinal fields at each space-time point over which we average - and (ii) clean circuits on qudits. In case (i), we rigorously show that there exists a class of generic (not finetuned) dual-unitary circuits that is "stable" under perturbations. More precisely, when these systems are perturbed, correlations continue to propagate along one-dimensional paths in the space-time, though these paths do not need to be straight anymore. As a result, all points of the causal light cone acquire nontrivial correlations. To find the correlations between any two points, one has to sum the contribution of all "skeleton diagrams," i.e., all allowed space-time paths connecting them. Although our rigorous result holds only in the limit of vanishing density of perturbed gates (or at fixed, but arbitrary, large order in perturbation theory), we provide further theoretical arguments and numerical evidence for its validity even when all gates are perturbed by a small enough perturbation. Moreover, in this setting, we discover four new types of circuits where the two-point correlation functions are exactly (without approximations) given by the sum of skeleton diagrams. Finally, we formulate two simple analytical conditions for identifying the stable subclass of dual-unitary circuits also in the clean case (ii). Considering qubit gates, we show numerically that the stable subclass exists and explicitly demonstrate that, for sufficiently small perturbation strength, the sum of skeleton diagrams approximates exact two-point correlation functions with arbitrary precision. Finally, we contrast these findings with the case of random perturbations, where the light-cone structure of dual-unitary dynamical correlations [11] is preserved (stable) and the only effect of the perturbations is an additional exponential damping along light rays.

Alongside the dynamics of unitary quantum circuits, we also discuss dynamics of a set of classical bistochastic many-body Markov chains, which can be written as local "Markov circuits." Indeed—as we show in the paper-the formal treatment of these two classes of systems is completely analogous. In particular, in Markov circuits, the property of "dual unitarity" is replaced by that of "dual bistochasticity," meaning that the circuit is bistochastic when propagating in both space and time directions. Remarkably, the average over random longitudinal fields discussed at point (i) above maps quantum unitary circuits into Markov circuits. Therefore, our aforementioned rigorous results find a direct application also in the case of interacting classical stochastic dynamics. 
The rest of the paper is structured as follows. In Sec. II, we describe the technical setting of the problem and introduce the necessary diagrammatic notation, while in Sec. III we present the basic strategy and the main results of the paper. In Sec. IV, we consider fixed perturbations in noisy or classical Markov systems. The validity of our conclusions for clean (nonrandom) perturbed dual-unitary circuits is demonstrated in Sec. V by combining analytical arguments with numerical results. Finally, Sec. VI contains our conclusions. Some further discussions, technical details, and proofs are relegated to the Appendixes.

\section{SETTING OF THE PROBLEM}

We consider one-dimensional many-body systems composed of $2 L$ qudits, each of them with $d$ internal states. In other words, we examine a chain where at each site there is a quantum system with Hilbert space $\mathcal{H} \simeq \mathbb{C}^{d}$ and denote an orthonormal basis of $\mathcal{H}$ by

$$
\mathcal{B}=\{|j\rangle, j=0, \ldots, d-1\} .
$$

The time evolution in this system is generated by discrete applications of "Floquet operators" $\mathbb{U}$ of the form

$$
\mathbb{U}=\bigotimes_{x \in \mathbb{Z}_{L}} U_{x, x+1 / 2} \bigotimes_{x \in \mathbb{Z}_{L}+1 / 2} U_{x, x+1 / 2}
$$

Here, we consider periodic boundary conditions, we assume translational invariance, and we represent diagrammatically the unitary operator $U_{x, x+1 / 2}=U$ ("local gate"), $x \in\{-L / 2, \ldots,-1 / 2,0,1 / 2,1, \ldots,(L-1) / 2\}$, as

$$
U=\neg .
$$

Finally, we adopt the convention of time running upward; namely, in a product $A B$, the symbol for the operator $B$ is depicted below $A$. Introducing the diagrammatic representation

$$
U^{\dagger}=\square
$$

we can express the unitarity of the gates in terms of the following diagrammatic rules:

$$
U U^{\dagger}=
$$

\section{A. Dynamical correlations in the folded picture}

In this work, we adopt the so-called "folded" representation of the circuit, a standard trick in tensor-network theory $[29,30]$ that has recently found many applications in the studies of local quantum circuits [5,13-15,22,23,31-33]. Here, we consider the simplest possible folding mapping that consists of turning operators into states of a quantum circuit with larger local Hilbert space by "bending" their lower legs on top of the upper ones. More specifically, the folding mapping turns operators in $\operatorname{End}\left[\left(\mathbb{C}^{d}\right)^{\otimes 2 L}\right]$ into states in $\left(\mathbb{C}^{d^{2}}\right)^{\otimes 2 L}$. For example, considering local operators and following exactly the notation of Ref. [13], we have

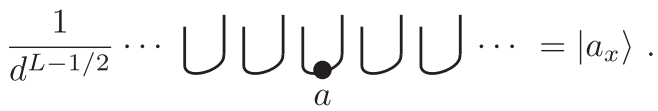

$$
\left|a_{x}\right\rangle=|\bigcirc\rangle^{\otimes(L+2 x)} \otimes|a\rangle \otimes|\bigcirc\rangle^{\otimes(L-1-2 x)} .
$$
are Hilbert-Schmidt normalized, which lead to normalized states. Moreover, introducing

$$
|O\rangle:=\frac{1}{\sqrt{d}} \bigcup \equiv d, \quad|a\rangle:=\bigsqcup_{a} \equiv \underset{a}{\downarrow_{a}},
$$

we can rewrite the rhs of Eq. (6) as
The folding mapping can also be applied to time-evolved operators in the Heisenberg picture. Considering again the example of local operators, we have 


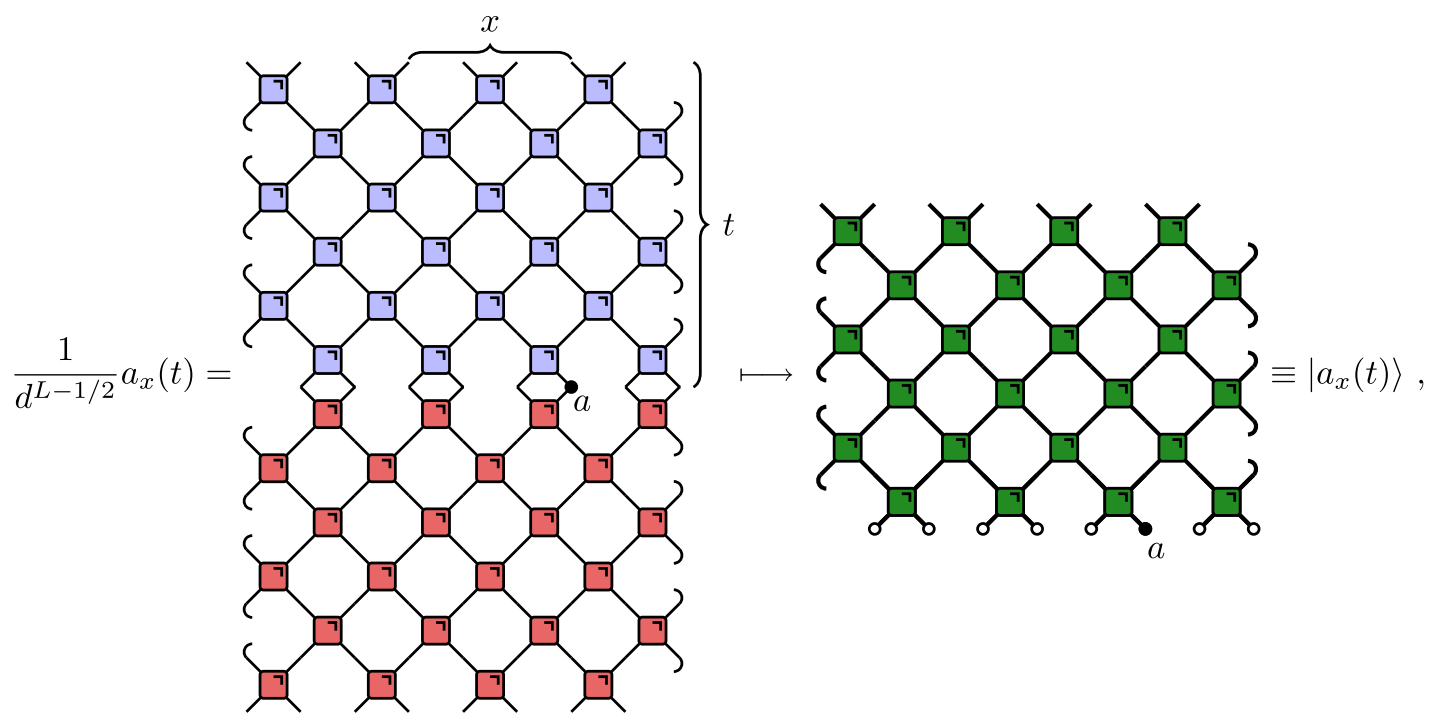

where we introduce the "doubled gate" (or the Heisenberg operator gate)

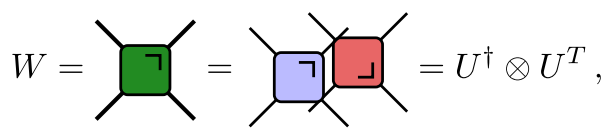

and denote by $(\cdot)^{T}$ the matrix transposition. Note that, on the level of the doubled gate, the relations (5) read as

$$
\sum_{0}=\left.\right|_{0}, \quad\{=?
$$

namely, they impose a unitality condition on $W$ ensuring that it maps the "identity state" $|\bigcirc \bigcirc\rangle$ into itself.

In this setting, we consider dynamical correlation functions in the infinite-temperature state, which in the folded picture are represented as

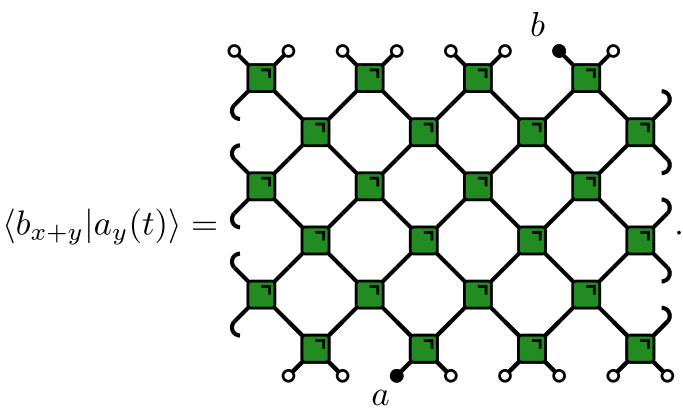

Whenever $t<2 L-|x+2 y|$, namely, when we are effectively in the thermodynamic limit, nontrivial correlations are contained in a causal light cone. This result is readily seen by using the rules (11), which allow us to rewrite (12) as follows:
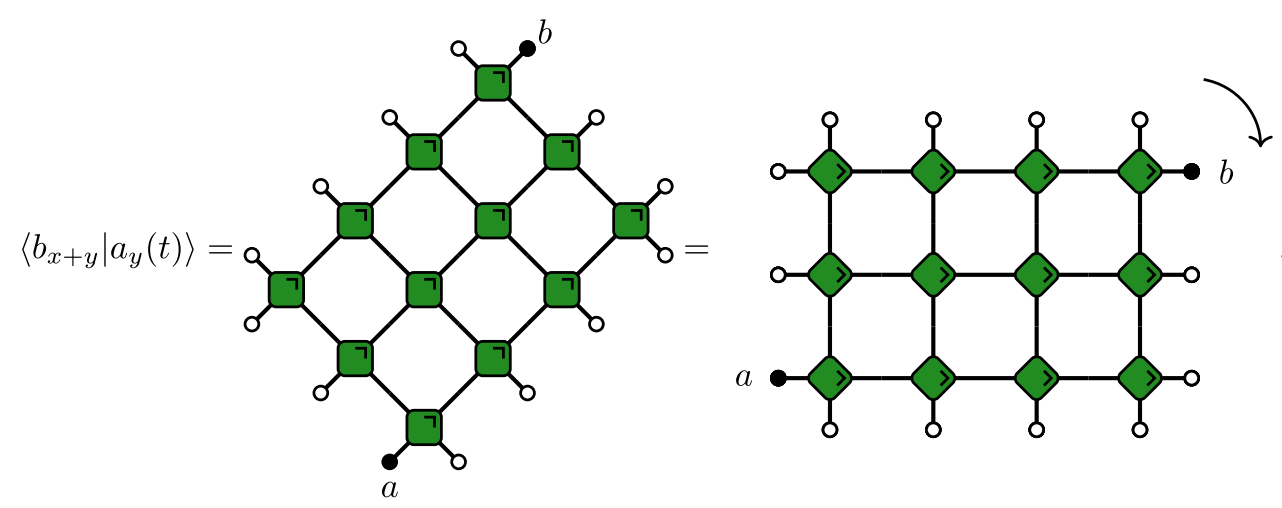
Here, we conveniently rotate the picture by $45^{\circ}$ in the clockwise direction, and, for concreteness, we depict correlation functions for integer coordinates $y \in \mathbb{Z}$ and $x \in \mathbb{Z}$. The cases with half-integer coordinates can be treated in an analogous fashion: If $y \in \mathbb{Z}+1 / 2$, the states at the bottom $(\bigcirc$ and $\bigcirc$ ) are exchanged, while when $x+$ $y \in \mathbb{Z}+1 / 2$ those at the top $(\bigcirc$ and $\bigcirc)$ are exchanged.

We see that in this representation correlation functions correspond to partition functions of a statistical mechanical model (with complex weights determined by the tensor $W$ ) defined on a rectangular lattice of dimensions

$$
\begin{array}{r}
x_{+}=t+\lceil x\rceil, \quad x_{-}=t+1-\lceil x\rceil, \quad y \in \mathbb{Z}, \\
x_{+}=t+1+\lceil x\rceil, \quad x_{-}=t-\lceil x\rceil, \quad y \in \mathbb{Z}+\frac{1}{2},
\end{array}
$$

where we introduce the ceiling function $\lceil\cdot\rceil$, such that $\lceil x\rceil \in \mathbb{Z}$ and $x \leq\lceil x\rceil<x+1$ for any $x \in \mathbb{R}$ (this definition applies also for negative $x$ ). Moreover, we note that for values of $x$ such that $x_{ \pm} \leq 0$ the correlations vanish identically. For definiteness, from now on, we always consider $y=0$ unless otherwise stated.

In the representation (13), it is natural to think of the correlations in terms of horizontal and vertical transfer matrices
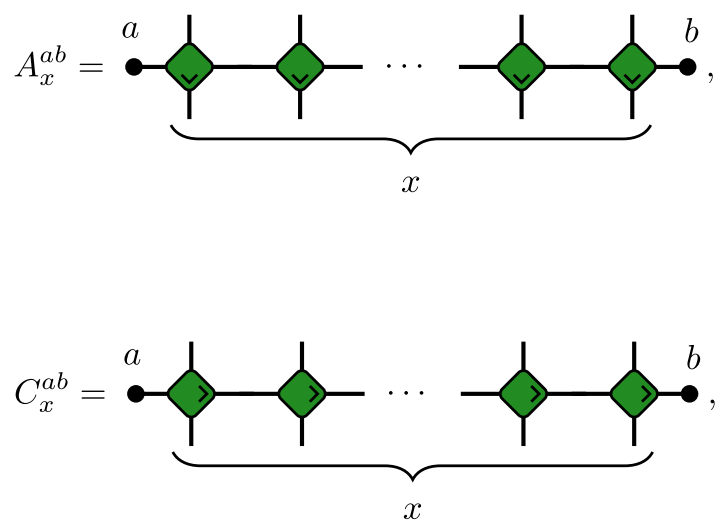

as follows:

$$
\left\langle b_{x} \mid a_{0}(t)\right\rangle= \begin{cases}\left\langle a \bigcirc \ldots \bigcirc\left|\left(A_{x_{-}}^{\bigcirc \bigcirc}\right)^{x_{+}-1} A_{x_{-}}^{\bigcirc b}\right| \bigcirc \ldots \bigcirc\right\rangle=\left\langle\bigcirc \ldots \bigcirc b\left|\left(C_{x_{+}}^{\circ \bigcirc}\right)^{x_{-}-1} C_{x_{+}}^{a \bigcirc}\right| \bigcirc \ldots \bigcirc\right\rangle, & x \in \mathbb{Z}+\frac{1}{2}, \\ \left\langle a \bigcirc \ldots \bigcirc\left|\left(A_{x_{-}}^{\bigcirc \bigcirc}\right)^{x_{+}}\right| \bigcirc \ldots \bigcirc b\right\rangle=\left\langle\bigcirc \ldots \bigcirc\left|C_{x_{+}}^{\bigcirc b}\left(C_{x_{+}}^{\circ \bigcirc}\right)^{x_{-}-2} C_{x_{+}}^{a \bigcirc}\right| \bigcirc \ldots \bigcirc\right\rangle, & x \in \mathbb{Z} .\end{cases}
$$

Note that the above transfer matrices fulfill the following two properties:

(i) $A_{x}^{a b}$ and $C_{x}^{a b}$ are contracting; i.e., their eigenvalues lie on the closed unit disk around 0 in the complex plane, for all $a, b$. This property is a consequence of the unitarity of $W$ and can be established following the derivation in Appendix A of Ref. [13].

(ii) The state $|\bigcirc\rangle^{\otimes x}$ is an eigenvector of $A_{x}^{\bigcirc \bigcirc}$ and $C_{x}^{\bigcirc \bigcirc}$ with eigenvalue one, which is a direct consequence of the unitality relations (11).

The folding mapping described in this subsection turns the evolution of operators in the quantum circuit defined by the elementary gate $U$ into that of states in a larger (super) quantum circuit defined by the elementary gate $W$. In this language, the correlation functions are nothing but matrix elements of powers of the evolution operator

$$
\mathbb{W}=\bigotimes_{x \in \mathbb{Z}_{L}+1 / 2} W_{x, x+1 / 2} \bigotimes_{x \in \mathbb{Z}_{L}} W_{x, x+1 / 2}
$$

between two specific states. In particular, those of local operators are matrix elements of $\mathbb{W}^{t}$ between two "oneparticle states" composed by the tensor product of $2 L-1$ copies of $|\bigcirc\rangle$ and one state orthogonal to it $[|a\rangle$ and $|b\rangle$ in Eq. (12)]. Matrix elements of this kind can be brought to the form (13). Even though the gate $W$ is unitary by construction [cf. Eq. (10)], the unitarity of $W$ is not needed for the simplification: One just needs the unitality property (11). Therefore, this setting can be used to study more general problems than that of computing correlations in unitary quantum circuits. An example of it is given in Appendix A, where we use it to study correlations in "Markov circuits," i.e., classical Markov chains where at each half time step the time evolution couples only nearest neighbors (in a brickwork fashion) with bistochastic matrices [see the definition in Eq. (A7)].

\section{B. Dual-unitary gates}

As observed in Ref. [11], the correlations drastically simplify whenever the gate, together with Eq. (11), also fulfills
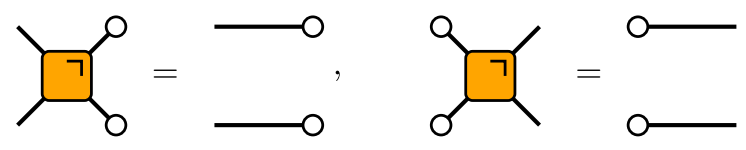

We use a different (orange) color to denote doubled gates fulfilling these two additional conditions. The conditions (20) originate from the following requirements on the single (unfolded) gates: 


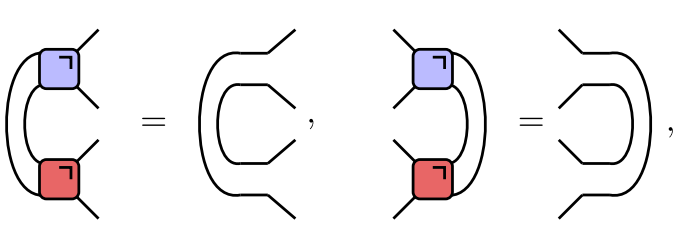

and essentially imply that the evolution of the system remains unitary when one exchanges the roles of space and time. Gates with these properties, called "dual unitary," have recently been used to obtain exact results in a number of different problems concerning nonequilibrium dynamics of quantum many-body systems and quantum many-body chaos [11-19].

The simplification of dynamical correlations can be seen, for instance, by applying the first of Eqs. (20) to the rightmost corner of Eq. (13):

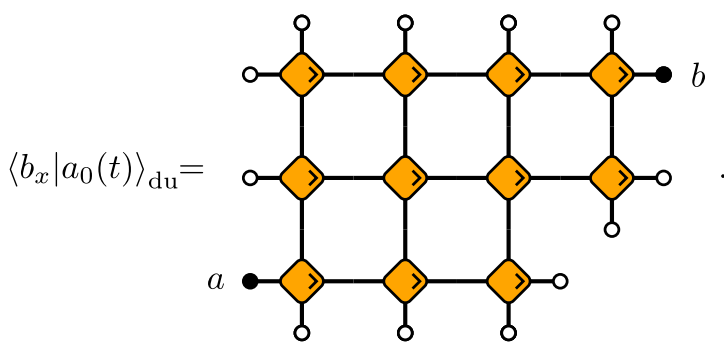

We see that the relation can be applied further and ultimately allows us to bring the diagram in the following form:

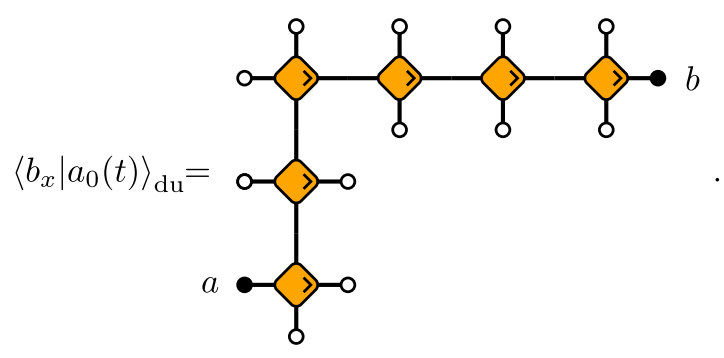

By applying the second of Eqs. (20), we see that the correlation factorizes and reduces to

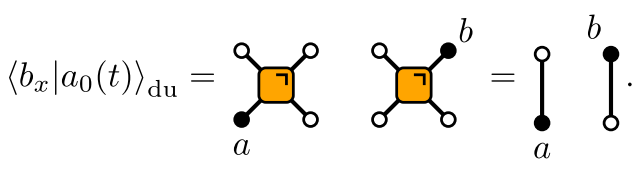

These two terms are zero for $a$ and $b$ orthogonal to the identity operator, i.e., if they are traceless. Following this derivation, it is easy to see that the only nontrivial correlations are obtained in diagrams with no corners with two neighboring $\bigcirc$, namely, when the rectangle in Eq. (13) reduces to a line with two bullets at the ends. For integer starting points, $y \in \mathbb{Z}$, this reduction happens when

$$
x=t \Rightarrow x_{+}=2 t, \quad x_{-}=1 .
$$

In this case, the diagram reads as

$$
\left\langle b_{x} \mid a_{0}(t)\right\rangle_{\mathrm{du}}=a \underbrace{2}_{0} \rightarrow b
$$

where in the last step we use the transfer matrix (16) for a simple one-dimensional system $(x=1)$. Moreover, we add the subscript "du" to stress that $A_{\mathrm{du}, x}^{\circ \circ}$ is made from dualunitary gates.

Analogously, when the starting point is a half-odd integer, say, $y=1 / 2$, the correlation is nonzero only for

$$
x=-t \Rightarrow x_{+}=1, \quad x_{-}=2 t
$$

and reads as

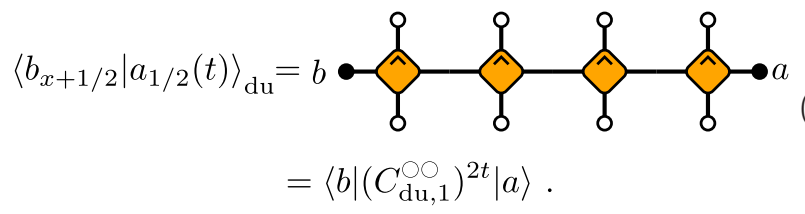

In summary, in dual-unitary circuits, the correlations are entirely determined by 1D transfer matrices (or, equivalently, one-qudit maps) and take the following simple form:

$$
\begin{aligned}
\left\langle b_{x+y} \mid a_{y}(t)\right\rangle_{\mathrm{du}}= & \bmod (2 y+1,2) \delta_{x-t}\left\langle a\left|\left(A_{\mathrm{du}, 1}^{\bigcirc \bigcirc}\right)^{2 t}\right| b\right\rangle \\
& +\bmod (2 y, 2) \delta_{x+t}\left\langle b\left|\left(C_{\mathrm{du}, 1}^{\circ \bigcirc}\right)^{2 t}\right| a\right\rangle,
\end{aligned}
$$

where $\delta_{x}$ denotes the Kronecker delta function and $\bmod (m, n) \equiv m \bmod n$ is the mod function. As discussed in Ref. [11], depending on the spectrum of the transfer matrices $A_{\mathrm{du}, 1}^{\circ \bigcirc}$ and $C_{\mathrm{du}, 1}^{\circ \bigcirc}$, these correlations can show four increasing degrees of ergodicity ranging from noninteracting behavior-where correlations are all constant - to the ergodic and mixing one-where all correlations decay exponentially. In particular, by providing a complete parametrization of dual-unitary gates for $d=2$, Ref. [11] shows that the ergodic and mixing case is typical (i.e., it has measure one in the parametrization space).

Finally, we stress that if the double gate is defined as in Eq. (10) and $U$ fulfills Eq. (21), then also $W$ and $W^{\dagger}$ fulfill an analogous diagrammatic relation. This relation is, however, not needed to obtain the results of this subsection. We need only the conditions (20), which we dub "dual unitality." Even though the two conditions are equivalent when the gate $W$ comes from a folded quantum circuit, Eq. (20) is less restrictive and can hold in a more general setting. For example, in Appendix A, we show that this 
property arises in Markov circuits where the local evolution matrix is bistochastic in both space and time directions.

\section{STRATEGY AND RESULTS}

The goal of this paper is to develop a perturbative expansion of correlation functions around the dual-unitary point. The idea is to consider circuits with a number of nondual-unitary gates $U_{\eta}$ composed of a dual-unitary term $U_{\mathrm{du}}$ and a non-dual-unitary correction. To have gates that are manifestly unitary, we consider perturbations of the multiplicative form

$$
U_{\eta}=U_{\mathrm{du}} e^{i \eta P},
$$

where $P$ is a generic Hermitian two-qudit operator and nonnegative real parameter $\eta$ sets the strength of the perturbation. The folded gate $W_{\eta}$ can then be written as

$$
W_{\eta}=\left(e^{-i \eta P} \otimes e^{i \eta P^{T}}\right) W_{\mathrm{du}} .
$$

Note that any quantum gate can be written in the form (30). Therefore, the expression (30) is the most general perturbation preserving the circuit structure of the time evolution.

When representing correlation functions as partition functions in a lattice of doubled gates [cf. Eq. (13)], one can think of the gates $W_{\eta}$, for $\eta>0$, as defects. Even though the methods that we develop are applicable to arbitrary distributions of defects, for the sake of clarity, in the main text we consider only the case where all defects are the same; namely, there is a nonrandom systematic breaking of dual unitarity. For comparison, we consider in Appendix $\mathrm{C}$ the opposite extreme case of random defects independently distributed at each space-time point. To better control the perturbation theory, it is useful to also modulate the number of defects in the lattice. To this aim, we introduce an additional parameter: the density $\delta$ of defects. We fix the density, because the actual arrangement of the defects does not affect appreciably the physics: One can imagine to randomly place $\delta x_{+} x_{-}$defects among the $x_{+} x_{-}$gates in the lattice (13). For simplicity, however, it is sometimes useful to imagine the defects covering a regular sublattice of Eq. (13) with lattice spacings $\nu_{+}$and $\nu_{-}$, such that $\delta=1 / \nu_{+} \nu_{-}$. For example,

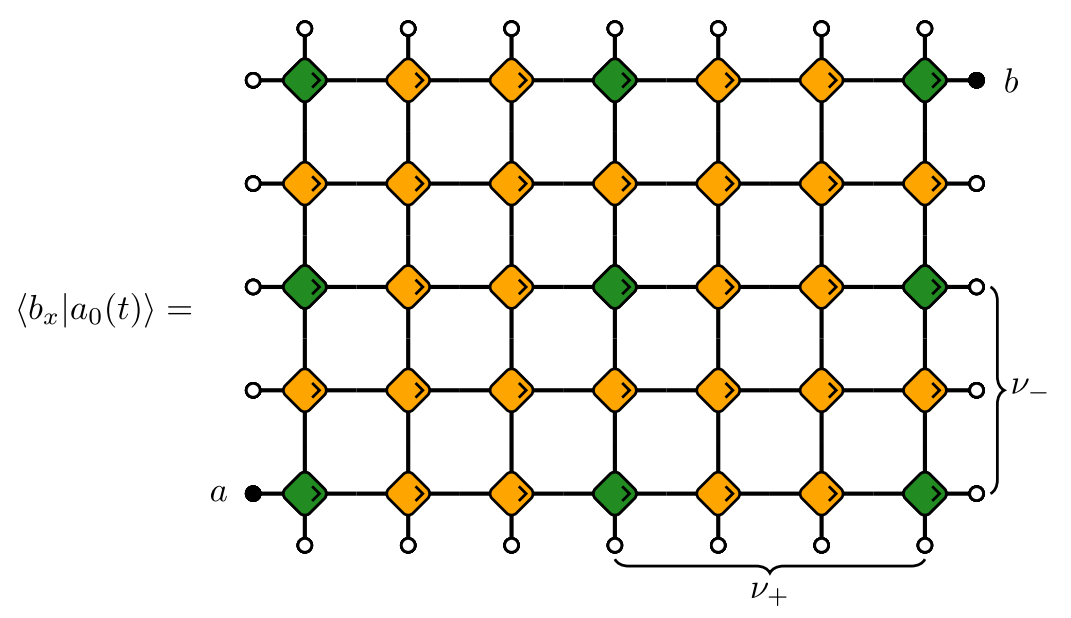

It follows directly from the above definitions that in both limiting cases, $\eta \rightarrow 0$ or $\delta \rightarrow 0$, we recover a dual-unitary circuit. The two perturbations, however, are highly inequivalent. In particular, the case of small density $\delta \ll 1$ is substantially easier to treat than that of small strength $\eta \ll 1$ and allows for rigorous results. This difference can be appreciated through a simple combinatoric argument: While for small $\delta$ one can work with a single partition function with a small number of defects and large dualunitary islands, separating the dual-unitary islands for nonzero $\eta$ generates a complicated sum of terms. In particular, the number of contributions at a given order $\eta^{n}$ corresponds to the number of ways to dispose $n$ identical objects in $x_{+} x_{-}$identical drawers and becomes exponentially large in the volume $x_{+} x_{-}$for large enough $n$.

Remarkably, in this paper, we find that-under certain conditions on the unperturbed dual-unitary gate $W_{\mathrm{du}}$-the leading-order contribution to the correlations can be directly computed in both cases and, surprisingly, takes the same form. Specifically, we observe that—at the leading order in time-correlations are still determined by the 1D transfer matrices $A_{\mathrm{du}, 1}^{\circ \bigcirc}$ and $C_{\mathrm{du}, 1}^{\circ \bigcirc}$ [cf. Eqs. (26)-(28)]. The difference is that, instead of being contracted along straight lines as in Eqs. (26) and (28), now the maps can also be contracted along zigzag lines like 


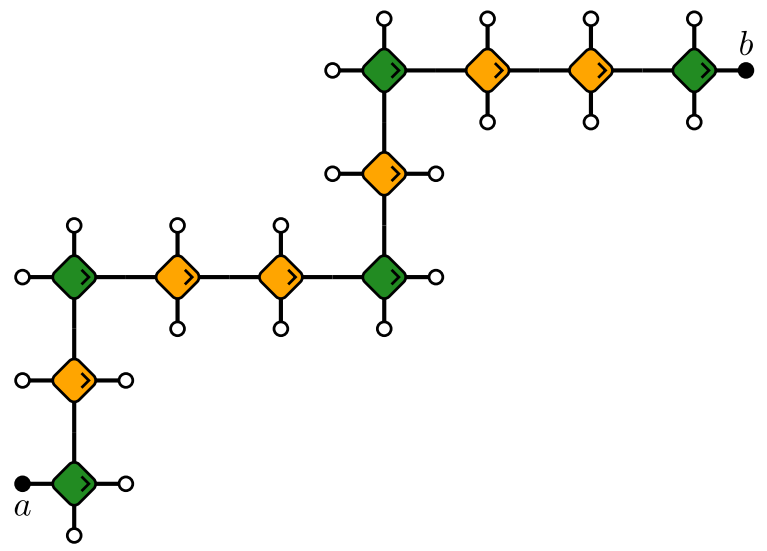

which we dub "skeleton diagrams." In particular, the correlation between two arbitrary points in the causal light cone is obtained by summing the contributions of all skeleton diagrams connecting the two points. The turns in the diagrams are generated by the defects, which means that for $\delta<1$ all the possible positions of the turns are restricted to a sublattice, while for $\delta=1$ the turns can be anywhere in the lattice. Note that all skeleton diagrams with down or left turns are forbidden. Indeed, these diagrams are cut by the rules (11) and do not contribute to the correlation. Such decomposition of the correlation function can be interpreted as a discrete path integral on the 2D lattice (32).

To explain how and under what conditions this simplification arises, we proceed in two steps. First, in Sec. IV, we present rigorous results in a toy setting where the wires in Eq. (32) are two dimensional and the doubled gates four dimensional-we dub this case "reduced gates." Second, in Sec. V, we use a combination of analytical arguments and numerical observations to show that the phenomenology identified in the toy setting holds true for generic $d^{4}$-dimensional doubled gates.

Let us summarize our results in the above two cases. At the end, for a comparison, we also provide a summary of the results we obtain for random, independently distributed perturbations (details are in Appendix C).

\section{A. Results on reduced gates}

We begin by noting that, since the doubled or folded gates have dimension $d^{4}$, one cannot reduce the dimension of these gates below 16 by lowering the local Hilbert space dimension $d$. However, as explained in Sec. IV, it is possible to reduce the problem by considering unperturbed dual-unitary gates with random $U(1)$ noise (describing, for example, random magnetic fields in a fixed direction for spin $1 / 2$ degrees of freedom, $d=2$ ) and focusing on the correlations averaged over the noise. In this case, each wire in Eq. (32) becomes effectively a two-state system, and we consider the following basis:

$$
\mathcal{B}=\{|\bigcirc\rangle,|\bigcirc\rangle\}
$$

where $|\bigcirc\rangle$ corresponds to the identity operator and $|\mathbf{O}\rangle$ is orthogonal to it (it corresponds to the only nontrivial onesite traceless operator preserved by the average). The most general averaged folded two-body gate can be expressed in the basis $\mathcal{B} \otimes \mathcal{B}$ as

$$
w:=\square=\left(\begin{array}{cccc}
1 & 0 & 0 & 0 \\
0 & p \varepsilon & a & b \\
0 & c & q \varepsilon & d \\
0 & e & f & g
\end{array}\right),
$$

where we use lowercase $w$ and thin lines to highlight that the wires are just two dimensional and the gate is, hence, four dimensional.

Since the gate $w$ is obtained via an average, it is not unitary. However, we see that Eq. (35) fulfills the conditions (11). Moreover, we see that the gate also fulfils the conditions (20) if we set $\varepsilon=0$, namely,

$$
w_{\mathrm{du}}:=\square=\left(\begin{array}{llll}
1 & 0 & 0 & 0 \\
0 & 0 & a & b \\
0 & c & 0 & d \\
0 & e & f & g
\end{array}\right) .
$$

The parameter $\varepsilon$ can then be interpreted as the dualunitarity breaking parameter in this setting. Indeed, expressing the elements of $w$ in terms of those of $W_{\eta}$ (see Appendix B 1), we find that $\varepsilon=\mathcal{O}(\eta)$, where $\eta$ is the strength of the perturbation in Eq. (31). On the other hand, all other parameters in $w$ are $\mathcal{O}\left(\eta^{0}\right)$. In words, this expression means that $\varepsilon$ vanishes for vanishing $\eta$, while all other parameters in $w$ are, in general, nonzero.

More generally, from the explicit parametrization in Appendix B 1, we also find that $w_{i j} \in[-1,1]$ and that the gate becomes a bistochastic matrix [see the definition (A7)] when conjugated with $H \otimes H$, where

$$
H:=\frac{1}{\sqrt{2}}\left(\begin{array}{cc}
1 & 1 \\
1 & -1
\end{array}\right)
$$

is the Hadamard transformation. As shown in Appendix A, this result means that the evolution generated by $w$ can be mapped to that of a Markov circuit defined on a chain of two-state systems. Remarkably, we also observe the opposite: Every local propagator of a Markov circuit on twostate systems can be mapped into a $U(1)$-averaged unitary gate. This observation means that all our results on reduced gates are also applicable to Markov circuits.

For reduced gates, all nontrivial correlations are proportional to $\left\langle\boldsymbol{O}_{x} \mid \boldsymbol{\Theta}_{0}(t)\right\rangle$, and we find the following three main results. 


\section{Exactly solvable cases}

Apart from the dual-unitary point $\varepsilon=0$, the gate (35) has four additional nontrivial exactly solvable points:
(i) $p=0$;
(ii) $q=0$;
(iii) $b=d=0$;
(iv) $e=f=0$;

where the parameters that are not set to zero can take arbitrary values. As shown in Sec. IV B, in all these cases the correlation functions are exactly given by the sum of skeleton diagrams. In particular, considering a regular sublattice of defects as in Eq. (32), we find

$$
\left\langle\boldsymbol{\bullet}_{x} \mid \boldsymbol{\Theta}_{0}(t)\right\rangle= \begin{cases}a^{x_{+}} \delta_{x_{-}-1}+\sum_{n=1}^{\bar{n}_{1}}\left(p q \varepsilon^{2}\right)^{n}\left(\begin{array}{c}
\tilde{x}_{+} \\
n
\end{array}\right)\left(\begin{array}{c}
\tilde{x}_{-}-2 \\
n-1
\end{array}\right) a^{x_{+}-n} c^{x_{-}-n-1} & x \in \mathbb{Z}, \\
q \varepsilon \sum_{n=0}^{\bar{n}_{2}}\left(p q \varepsilon^{2}\right)^{n}\left(\begin{array}{c}
\tilde{x}_{+}-1 \\
n
\end{array}\right)\left(\begin{array}{c}
\tilde{x}_{-}-1 \\
n
\end{array}\right) a^{x_{+}-n-1} c^{x_{-}-n-1} & x \in \mathbb{Z}+1 / 2,\end{cases}
$$

where we introduce the integers $\bar{n}_{1}=\min \left(\tilde{x}_{+}, \tilde{x}_{-}-1\right)$ and $\bar{n}_{2}=\min \left(\tilde{x}_{-}-1, \tilde{x}_{+}-1\right)[34]$ and the rescaled light-cone coordinates

$$
\tilde{x}_{ \pm}=\left\lfloor x_{ \pm} / \nu_{ \pm}\right\rfloor .
$$

Here, the symbol $\lfloor\cdot\rfloor$ denotes the floor function, such that $\lfloor x\rfloor \in \mathbb{Z}$ and $x-1<\lfloor x\rfloor \leq x$ for any $x \in \mathbb{R}$.

Note that the ray along which Eq. (38) exhibits the slowest asymptotic decay in time is generically different from the light ray (i.e., the unit speed ray) [35]; a representative example is reported in Fig. 1.

We stress that, generically, the gates fulfilling either of (i)-(iv) generate highly complex dynamics; e.g., they efficiently scramble quantum information. For example, we numerically computed a standard dynamical complexity indicator for locally interacting systems - the so-called local-operator entanglement [13,14,31,36-41]—observing a linear growth. The key property leading to the simple form (38) is that the dynamics generated by the gates (i)-(iv) are not time-reversal symmetric. In particular, it is true that evolving forward (backward) in time the support of local operators can grow, forming larger and larger strings of local operator products. Yet these large strings cannot shrink back and do not contribute to the overlap with ultralocal operators. The fact that very large strings do not contribute much

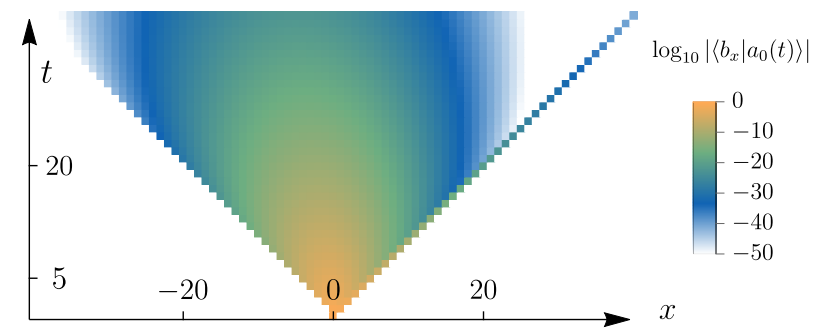

FIG. 1. Correlations between integer sites given by Eq. (38). The correlations are nonzero within the whole light cone, and the slowest decay is along $\left|\zeta^{*}\right|<1$. We use defects at all sites $(\delta=1)$ and parameters $\varepsilon=0.4, p=q=1, a=0.0945626$, and $c=$ 0.195892 (as in gate 3 in Table I). to the correlations of local operators is expected to hold quite generally. For example, a similar idea has recently been invoked in Ref. [5] to devise a numerical method able to access the late-time regime. The key point is that this property becomes exact in cases (i)-(iv).

\section{Low-density limit}

Two simple conditions on the spectrum of horizontal and vertical transfer matrices constructed with the reduced dualunitary gate (36) (see Sec. IV C 1) allow us to prove that the expression (38) is the dominant contribution to the correlation function at low density $\delta$ and for any $\varepsilon$. More precisely, if the horizontal (vertical) transfer matrix fulfills the aforementioned conditions, Eq. (38) is dominant in the limit

$$
x_{+(-)}, \bar{\nu}_{+(-)} \rightarrow \infty, \quad \tilde{x}_{+(-)}=\text {fixed },
$$

where $\bar{\nu}_{+(-)}$is the minimal separation among the defects in the horizontal (vertical) direction and the relative error decays exponentially in $\bar{\nu}_{+(-)}$. In Sec. IV C 1 , we prove that these conditions hold if the parameters of the gate $w_{0}$ [cf. Eq. (36)] fulfill

$$
|a|>a^{2}+\frac{|b f|}{1-\alpha} \quad \text { or } \quad|c|>c^{2}+\frac{|d e|}{1-\beta},
$$

where $\alpha$ and $\beta$, respectively, denote the largest singular values of the submatrices

$$
\left(\begin{array}{ll}
c & e \\
d & g
\end{array}\right) \text { and }\left(\begin{array}{ll}
a & f \\
b & g
\end{array}\right)
$$

As explained in Sec. IV [see, in particular, the discussion around Eq. (79)], these conditions ensure that correlations propagate on paths of width 1 .

\section{Small strength at density one}

Based on the rigorous results described in Sec. III A 2, we argue that skeleton diagrams give the dominant contribution also for $\delta=1$ and $\varepsilon \ll 1$ if both conditions (41) 
are satisfied. As explained in Sec. IV C 2, the main idea is that, even though Eq. (38) does not provide a complete perturbative expansion in $\varepsilon$, at each fixed order in perturbation theory the skeleton diagrams dominate for $x_{+}$and $x_{-}$ both large. This argument is tested in Sec. IV C 2 by comparing Eq. (38) with the exact correlations evaluated numerically: The observed agreement is excellent. An example of the correlation pattern described by Eq. (38) in this case is depicted in Fig. 2.

\section{B. Results on generic gates}

Considering quantum gates acting on qudits of generic dimension $d$ (and again focusing on a regular sublattice of defects), the contribution of skeleton diagrams reads as

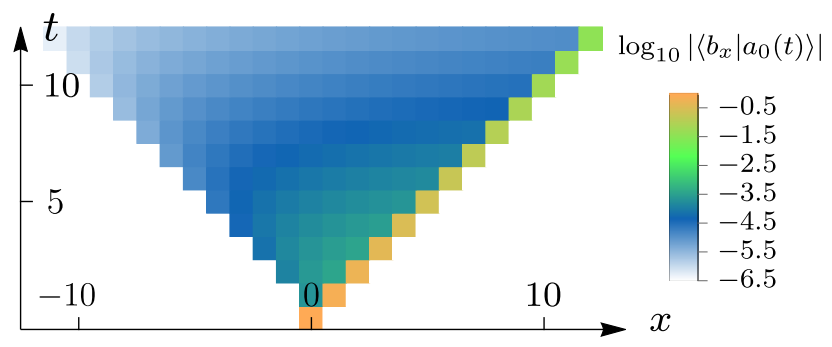

FIG. 2. Correlations between integer sites in a perturbed dualunitary circuit with density of defects $\delta=1$, where the gate is defined in Table I (gate 2). We see a reminiscence of the dualunitary behavior with a strong peak along the light-cone edge. As opposed to the pure dual-unitary result, however, the correlations are nonzero within the whole light cone.

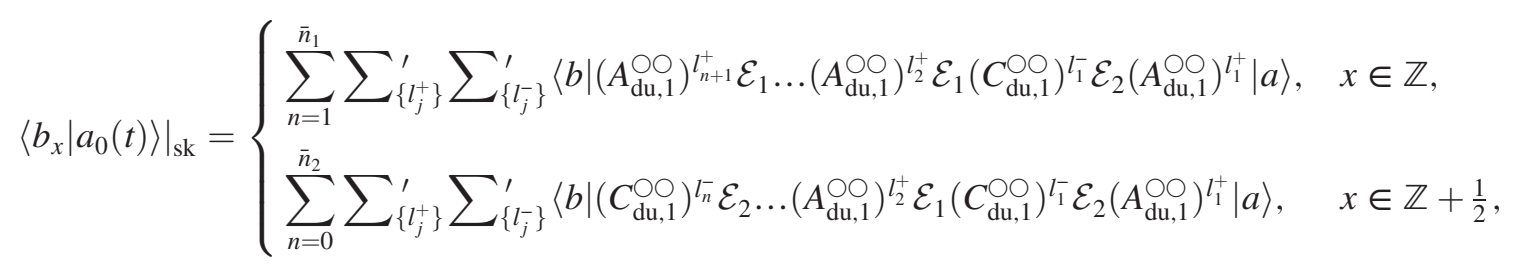

where $\quad \bar{n}_{1}=\min \left(\tilde{x}_{+}, \tilde{x}_{-}-1\right) \quad$ and $\quad \bar{n}_{2}=\min \left(\tilde{x}_{-}-1\right.$, $\left.\tilde{x}_{+}-1\right)$. We denote by
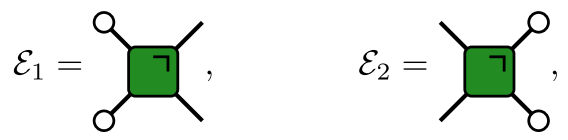

the "defect maps," and the primed sums are subject to the constraints

$$
\begin{aligned}
& \sum_{j=1}^{n+1} l_{j}^{+}=x_{+}-n, \quad \sum_{j=1}^{n} l_{j}^{-}=x_{-}-n-1, \quad x \in \mathbb{Z}, \\
& \sum_{j=1}^{n} l_{j}^{+}=x_{+}-n, \quad \sum_{j=1}^{n} l_{j}^{-}=x_{-}-n, \quad x \in \mathbb{Z}+\frac{1}{2} .
\end{aligned}
$$

The expression (43) is a direct generalization of Eq. (38), where one replaces numbers $a, c, p \varepsilon$, and $q \varepsilon$ with $d^{2} \times d^{2}$ matrices $A_{\mathrm{du}, 1} \mathrm{O}, C_{\mathrm{du}, 1}^{\bigcirc \bigcirc}, \mathcal{E}_{1}$, and $\mathcal{E}_{2}$, respectively.

The conditions on the spectrum of horizontal and vertical transfer matrices mentioned in Sec. III A 2 are sufficient to rigorously prove the dominance of skeleton contributions at low densities also in the generic case (where the matrices are constructed with nonreduced dual-unitary gates). Although we cannot analytically determine the family of gates $W_{\mathrm{du}}$ for which the conditions are fulfilled, we numerically identify such a family for quantum circuits of qubits $(d=2)$ in Sec. V. For gates in this family, the argument discussed in Sec. III A 3 remains valid as well. Accordingly, we find that if $x_{+}$and $x_{-}$are both large, Eq. (43) gives the most relevant contribution to correlations also for $\delta=1$ and $\eta \ll 1$.
Let us now comment on the physical content of Eq. (43). The sum is composed of powers of one-dimensional maps. These maps typically have nontrivial eigenvalues strictly smaller than one, resulting in exponential decay of each term in the sum. Therefore, also the sum of all terms generically decays exponentially in $t$. For some special choices of the parameters, however, the exponent of the decay can approach 0 . For instance, if the circuit has a local conserved charge, e.g., magnetization, we expect diffusive correlations that decay with time as $\propto 1 / t$. This result is, in principle, allowed by Eq. (43).

\section{Uncorrelated random perturbations}

Systematic defects (homogeneous in the space-time) have a much more drastic effect on the structure of correlations than random ones. Indeed, averages over uncorrelated random defects (30) (with $P$ distributed according to the Gaussian unitary ensemble) preserve the dual-unitary form of correlations. The only effect of the defects is an additional damping factor that causes or enhances - depending on the degree of ergodicity of the unperturbed dual-unitary circuit (see the discussion at the end of Sec. II B) - the exponential decay of the correlations along the light-cone edge (light ray). Specifically, we have

$$
\begin{aligned}
& \mathbb{E}_{\mathrm{GUE}}\left[\left\langle b_{x+y} \mid a_{y}(t)\right\rangle\right] \\
& =\bmod (2 y, 2) \delta_{x+t}\left\langle b\left|\left(C_{\mathrm{du}, 1}^{\circ \circ}\right)^{2 t}\right| a\right\rangle\left[\frac{K_{\mathrm{GUE}}(\eta)-1}{d^{4}-1}\right]^{\tilde{x}_{-}} \\
& +\bmod (2 y+1,2) \delta_{x-t}\left\langle a\left|\left(A_{\mathrm{du}, 1}^{\bigcirc \bigcirc}\right)^{2 t}\right| b\right\rangle\left[\frac{K_{\mathrm{GUE}}(\eta)-1}{d^{4}-1}\right]^{\tilde{x}_{+}},
\end{aligned}
$$


where the transfer matrices $A_{\mathrm{du}, 1}^{\circ \bigcirc}$ and $C_{\mathrm{du}, 1}^{\circ \bigcirc}$ are defined as in Eqs. (16) and (17) but in terms of the unperturbed dualunitary gate $W_{\mathrm{du}}$, the rescaled variables light-cone coordinates are defined in (39), and

$$
K_{\mathrm{GUE}}(x) \equiv \mathbb{E}_{\mathrm{GUE}}\left[\left|\operatorname{tr}\left[e^{i x P}\right]\right|^{2}\right]
$$

is the spectral form factor of the Gaussian unitary ensemble ( $\mathbb{E}_{\mathrm{GUE}}[\cdot]$ is the ensemble average).

An interesting point to note is that Gaussian defects with infinite strength allow for nonzero correlations; indeed,

$$
\lim _{\eta \rightarrow \infty} K_{\mathrm{GUE}}(\eta)=d^{2}
$$

This result should be compared with Haar random defects, which, instead, trivialize all correlations; i.e., the averaged correlation function becomes Kronecker delta in space-time for any positive density $\delta>0$ (see Appendix C). The two averages agree only in the $d \rightarrow \infty$ limit.

\section{REDUCED GATES}

In this section, we study the problem outlined in Sec. III considering the minimal setting where the wires in Eq. (32) are effectively two dimensional.

We begin considering the quantum circuit defined in Sec. II in the following special case.

(i) Fix $d=2$ and focus on unitary gates (3) of the form

$$
\bar{U}=\left(e^{i \phi_{1} \sigma^{z}} \otimes e^{i \phi_{2} \sigma^{z}}\right) \cdot U \cdot\left(e^{i \phi_{3} \sigma^{z}} \otimes e^{i \phi_{4} \sigma^{z}}\right),
$$

where $U$ is a generic $U(4)$ matrix, $\sigma^{z}$ is a Pauli matrix, and the phases $\phi_{1}, \phi_{2}, \phi_{3}$, and $\phi_{4}$ are independent random variables uniformly distributed in $[0,2 \pi]$. Once again, the random variables $\left\{\phi_{j}\right\}$ at each gate or space-time point are considered independent.

(ii) Consider two-point correlations averaged with respect to all $\left\{\phi_{j}\right\}$. This case corresponds to averaging over the single-site Haar measure of the $U(1)$ group. On the level of the folded gate $W$ [cf. Eq. (10)], the average corresponds to a simple projection, i.e.,

$$
\begin{aligned}
W \longmapsto w & =\mathbb{E}_{\left\{\phi_{j}\right\}}[W] \\
& =\left(P_{z} \otimes P_{z}\right) W\left(P_{z} \otimes P_{z}\right),
\end{aligned}
$$

where

$$
P_{z}:=|\mathbb{1}\rangle\left\langle\mathbb{1}|+| \sigma^{z}\right\rangle\left\langle\sigma^{z}\right| .
$$

In other words, the average over the Haar $U(1)$ measure projects each wire onto the subspace spanned by the diagonal matrices $\{|\bigcirc\rangle \equiv|\mathbb{1}\rangle,|\mathbf{O}\rangle \equiv$ $\left.\left|\sigma^{z}\right\rangle\right\}$ [cf. Eq. (7)], and, from now on, we conveniently use this reduced basis notation. In this basis the averaged two-qubit folded gates are effectively $4 \times 4$ matrices (while they are $16 \times 16$ without the average).

We see that, even though the $U(1)$ average simplifies the problem by reducing the size of the relevant local Hilbert space, it does not trivialize the two-folded gate and, in turn, the two-point correlations. This result is in contrast to most averages over Haar random unitary gates that are considered in recent literature [22-24,31,32,4261]: In the latter cases, the average of the doubled gate $W$ is trivial [as in Eq. (C14)] and so are two-point functions. The minimal folded gate with a nontrivial average is the one composed of four copies of the time evolution operator (two copies of $U$ and two copies of its Hermitian conjugate $\left.U^{\dagger}\right)$.

To be more specific, let us count the number of free parameters in the gate (51). This count can be conveniently done using the following parametrization for the generic unitary $U$ [cf. Eq. (50)] [62,63]:

$$
U=e^{i \phi}\left(u_{1} \otimes u_{2}\right) V\left[\left\{J_{j}\right\}\right]\left(u_{3}^{\dagger} \otimes u_{4}^{\dagger}\right) .
$$

Here, $\phi \in \mathbb{R}$,

$V\left[\left\{J_{j}\right\}\right]=\exp \left[i\left(J_{1} \sigma^{x} \otimes \sigma^{x}+J_{2} \sigma^{y} \otimes \sigma^{y}+J_{3} \sigma^{z} \otimes \sigma^{z}\right)\right]$,

and $u_{1}, u_{2}, u_{3}$, and $u_{4}$ are elements of $S U(2)$ in the fundamental representation. They can be expressed in terms of Euler angles

$$
u_{j}=e^{i\left(\alpha_{j} / 2\right) \sigma^{z}} e^{i\left(\beta_{j} / 2\right) \sigma^{y}} e^{i\left(\gamma_{j} / 2\right) \sigma^{z}} .
$$

From this parametrization, it is easy to see that the averaged folded gate (51) depends on 11 angles: two Euler angles $\left(\beta_{j}\right.$ and $\gamma_{j}$ ) for each single-wire unitary $u_{j}$ and the parameters $J_{1}, J_{2}$, and $J_{3}$. The explicit form of the averaged gate in the basis (34) is given by Eq. (35), where the explicit parametrization of the parameters $p \varepsilon, a, \ldots, g$ in terms of the above angles is given in Appendix B.

It is important to stress that the averaging procedure described above can be carried on for any fixed value of the angles $\left\{\beta_{j}, \gamma_{j}, J_{j}\right\}$. In particular, setting $J_{1}=J_{2}=\pi / 4$, one averages over gates in the dual-unitary subclass [11]. In this specific case, the averaged gate, now depending on seven parameters (see Appendix B), takes the form (36). Comparing with Eq. (35), we see that the effect of requiring dual unitarity is to set $\varepsilon$ to zero. Note that after the averaging the gate continues to fulfill the dual-unitality conditions (20).

Before turning to the analysis of correlation functions, we make a final remark. As shown in Appendix A, one can produce gates of the form (35) and (36) considering (dual-) bistochastic gates conjugated with $H \otimes H$ [cf. Eq. (37)]. Interestingly, there is a one-to-one correspondence between averaged (dual-)unitary gates and (dual-)bistochastic gates. 
Namely, given a legitimate averaged (dual-)unitary gate, one can produce a legitimate (dual-)bistochastic gate by conjugating it with $H \otimes H$ and vice versa. This result can be established numerically using the explicit parameterizations reported in Appendix B.

\section{A. Correlation functions as sums over paths}

Let us now examine Eq. (12) in the minimal setting under consideration in the section: two-dimensional wires and gates of the form (35). Inserting a resolution of the identity $\mathbb{1}=|O\rangle\langle O|+| \mathbf{O}\rangle\langle\mathbf{O}|$ at each wire, we can explicitly decompose Eq. (12) into the sum of $2^{x_{+} x_{-}}$terms. Each term admits a simple interpretation as a (weighted) path on the two-dimensional space or, analogously, as the spatial configuration of a certain polymer. To see this interpretation, let us introduce a different diagrammatic representation of the weights in terms of "tiles" where we connect O's with solid lines and ignore $\bigcirc$ 's. For example,

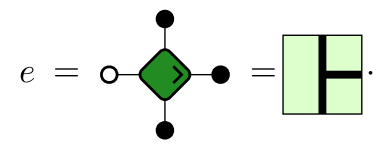

The complete set of tiles [corresponding to nonzero coefficients of the gate (35)] is given by

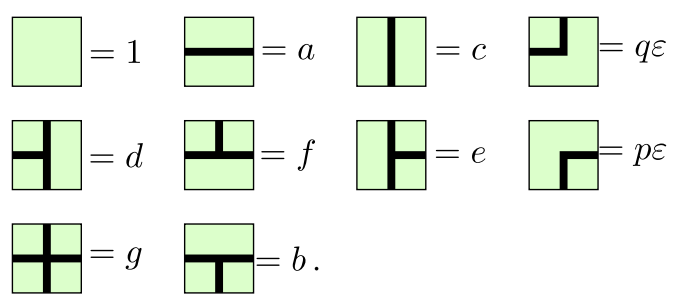

Then, the correlation function is expressed as

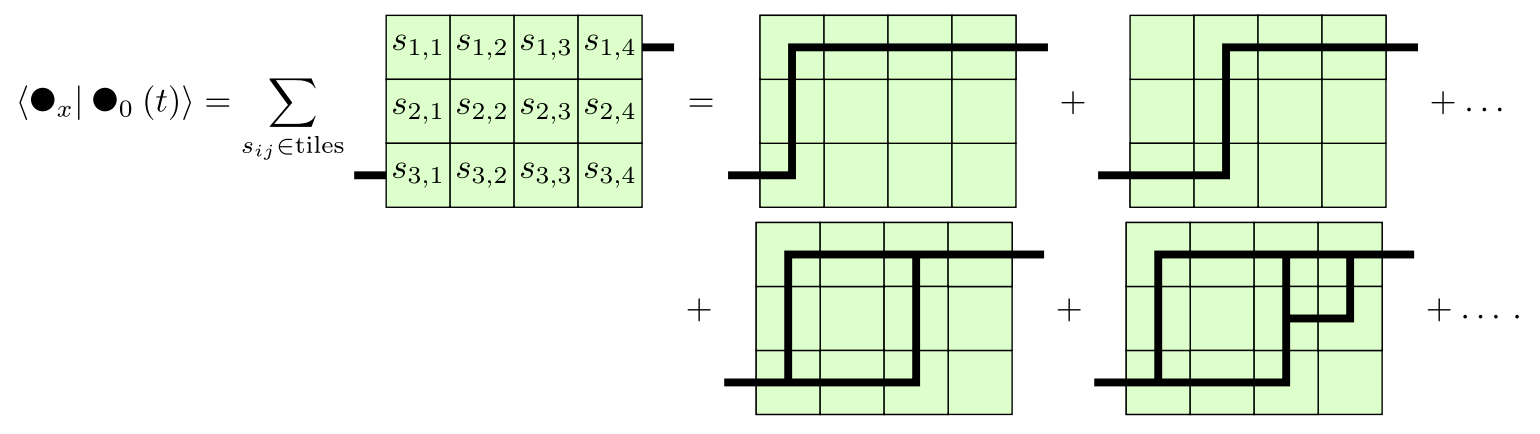

We use that all nontrivial states (i.e., those orthogonal to the "identity" $|\bigcirc\rangle$ ) are proportional to $|\mathbf{O}\rangle$. Namely,

$$
\left\langle b_{x} \mid a_{0}(t)\right\rangle \propto\left\langle\boldsymbol{\bullet}_{x} \mid \boldsymbol{\bullet}_{0}(t)\right\rangle,
$$

for all $a, b$ such that $\operatorname{tr}[a]=\operatorname{tr}[b]=0$. Moreover, in writing Eq. (58), we consider the case of $x, y \in \mathbb{Z}$. The other three possibilities correspond to different positions of the initial and final lines. Specifically, for $y \in \mathbb{Z}+1 / 2$ the initial line enters the bottom left tile from below, and for $x+y \in$ $\mathbb{Z}+1 / 2$ the final line exits the top right tile from above.

As suggested by the diagrammatic representation in Eq. (58), the correlation function can be thought of as the sum of paths with fixed end points, where different configurations have different (possibly negative) associated weights. The paths are allowed to split and merge, with weights $e, f$ and $b, d$, respectively, but they cannot "jump": Dangling ends of lines inside the diagram are forbidden. Finally, since downward and leftward turns are forbidden [cf. Eq. (57)], the paths can form loops only if they split [see, e.g., the last two diagrams on the rhs of Eq. (58)].

\section{B. Exactly solvable cases}

By inspecting the set of allowed tiles (57), we identify four nontrivial solvable cases, which we analyze in the following two subsections.

\section{Almost dual-unitary cases}

As observed before, the case $\varepsilon=0$ corresponds to the dual-unitary point and all correlations propagate along straight paths, with no turns allowed. Depending on the initial conditions (i.e., on whether $y$ is integer or half-odd integer), the straight lines are going either upward or rightward. This result is simply a path-sum reformulation of the general dual-unitary result (29) (cf. Ref. [11]).

Remarkably, however, the correlations remain exactly solvable also when only one of $p \varepsilon$ or $q \varepsilon$ vanishes. Indeed, in this case, we allow for only paths with a single turn and the rules (57) do not permit any "dressing," i.e., any thickening of the lines due to loops (see Sec. IV C 1 for a more precise definition). For example, choosing $p=0$ and $q \varepsilon \neq 0$, the correlations take the form given in Eq. (38), where only the first terms in each of the two lines can be nonzero [depicted in Eq. (82)]. We see that, apart from the 
straight paths described above, Eq. (38) establishes nontrivial correlations between integer and half-odd integer points.

\section{Non-dual-unitary solvable cases}

Surprisingly, the tiles (57) contain another nontrivial solvable limit that is not dual unitary. Indeed, there can be no loops in the paths whenever the "split weights" $f$ and $e$ or the "merge weights" $b$ and $d$ are zero. Consequently, when $(e, f)=(0,0)$ or $(b, d)=(0,0)$, the only allowed paths are the skeleton diagrams described in Sec. III [see, e.g., the first two diagrams on the rhs in Eq. (58)]. In this case, we can directly evaluate the correlation function (58) by summing all skeleton diagrams. In particular, if the defects cover a regular sublattice as in Eq. (32), one obtains the expression (38).

The formula (38) can be derived by straightforward combinatorics. Let us detail its derivation considering the case $x \in \mathbb{Z}$ as an example. We begin by noting that, since the path goes from the bottom left corner to the upper right one, it must involve the same number $n$ of right and up turns, with weights, respectively, given by $p \varepsilon$ and $q \varepsilon$. The minimum number of such turns is 0 (it contributes only for $x_{-}=1$ ), while the maximum, $\min \left(\tilde{x}_{+}, \tilde{x}_{-}-1\right)$, is set by the size of the defect's sublattice [the rescaled light-cone coordinates $\tilde{x}_{ \pm}$are defined in Eq. (39)]. For each fixed $n$, one has $n$ turns, $x_{+}-n$ horizontal segments, contributing with a factor $a^{x_{+}-n}$, and $x_{-}-1-n$ vertical ones, contributing with a factor $c^{x_{-}-1-n}$. To count all the possible ways in which the elementary pieces can be combined, we can consider the horizontal and the vertical directions separately. In the horizontal direction, we need to distribute $n$ indistinguishable pairs of turns (first up and then right) in $\tilde{x}_{+}$positions, leading to the combinatorial factor

$$
\left(\begin{array}{c}
\tilde{x}_{+} \\
n
\end{array}\right) \text {. }
$$

In the vertical direction, we are instead more constrained. Indeed, the first and last turns must be in the first and last row, respectively. The other $n-1$ pairs can be distributed freely in the remaining $\tilde{x}_{-}-2$ positions, leading to

$$
\left(\begin{array}{c}
\tilde{x}_{-}-2 \\
n-1
\end{array}\right)
$$

Putting it all together, we obtain the desired result. For defects on irregular sublattices, the reasoning is similar, but one has different combinatorial coefficients depending on the actual shape of the sublattice.

\section{Perturbation theory around the dual-unitary point}

Let us now consider the case of circuits that are perturbed away from the dual-unitary point. Namely, we consider the setting introduced in Sec. III: Among the $x_{+} x_{-}$gates in the lattice (13), there are $(1-\delta) x_{+} x_{-}$dual-unitary gates and $\delta x_{+} x_{-}$perturbations (or defects) breaking dual unitarity [see the pictorial representation in Eq. (32)]. As discussed in Sec III, we use two parameters to control the perturbations: strength $\varepsilon$ [which is proportional to $\eta$ in Eq. (31) for small $\eta$ ] and density $\delta$. We begin by considering the case of perturbation in the density of defects, which allows for a more rigorous analysis. Later, we see that, surprisingly, most of the rigorous conclusions drawn in that case apply also for small $\varepsilon$ and arbitrary $\delta \leq 1$.

\section{Low density, unit strength}

We begin our analysis by focusing on fixed defects with arbitrary strength $\varepsilon$ placed on a regular sublattice as in Eq. (32). In this case, there are regular strips (vertical and horizontal) composed only of dual-unitary gates. Whenever the widths $\nu_{ \pm}$[cf. Eq. (32)] of these strips become large enough, we can simplify the contribution by considering only the leading eigenvectors of the strips' transfer matrices $a_{\mathrm{du}, x}^{\circ \bigcirc}$ and $c_{\mathrm{du}, x}^{\circ}$. These are defined as in Eqs. (16) and (17) but using the $4 \times 4$ dual-unitary (or, rather, dual-bistochastic) gate $w_{\mathrm{du}}$ [cf. Eq. (36)], namely,
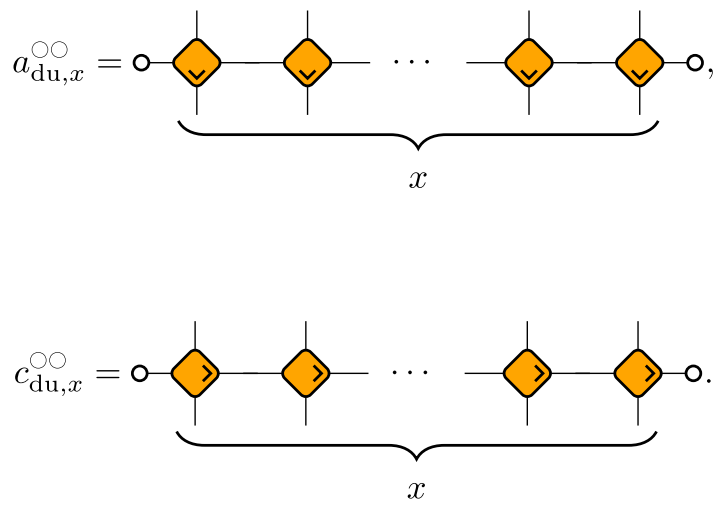

To treat these matrices, we make use of the following rigorous result, proved in Appendix D.

Property 1.- The matrices $a_{\mathrm{du}, x}^{\circ \bigcirc}$ and $c_{\mathrm{du}, x}^{\circ \bigcirc}$ take the following block diagonal form:

$$
\begin{aligned}
& a_{\mathrm{du}, x}^{\bigcirc \bigcirc}=p_{x, 0}^{\bigcirc}+a \sum_{k=1}^{x} p_{x, k}^{\bigcirc}+r_{1, x}, \\
& c_{\mathrm{du}, x}^{\bigcirc \bigcirc}=p_{x, 0}^{\bigcirc}+c \sum_{k=1}^{x} p_{x, k}^{\bigcirc}+r_{2, x},
\end{aligned}
$$

where we define

$$
\begin{aligned}
p_{x, 0}^{\bigcirc} & :=|\bigcirc\rangle^{\otimes x}\left\langle\left.\bigcirc\right|^{\otimes x},\right. \\
p_{x, k}^{\bigcirc} & :=|\underbrace{\bigcirc \ldots \bigcirc \bigcirc}_{k} \bigcirc \ldots \bigcirc\rangle\langle\underbrace{\bigcirc \ldots \bigcirc}_{k} \bigcirc \ldots \bigcirc|,
\end{aligned}
$$


and the "reminders" $r_{1, x}$ and $r_{2, x}$ are nonzero only in the subspaces defined by the projectors $\mathbb{1}-\sum_{j=0}^{x} p_{x, j}^{\bigcirc}$. Moreover,

$$
\left\|r_{1, x}\right\| \leq a^{2}+\frac{|b f|}{1-\alpha}, \quad \text { for }|a|>a^{2}+\frac{|b f|}{1-\alpha},
$$

and analogously

$$
\left\|r_{2, x}\right\| \leq c^{2}+\frac{|d e|}{1-\beta}, \quad \text { for }|c|>c^{2}+\frac{|d e|}{1-\beta},
$$

where $\alpha, \beta \in[0,1]$ are, respectively, the operator norms (largest singular values) of the matrices

$$
\left(\begin{array}{ll}
c & e \\
d & g
\end{array}\right), \quad\left(\begin{array}{ll}
a & f \\
b & g
\end{array}\right) .
$$

Consider now a vertical strip $x_{-} \times \nu_{+}$with horizontal transfer matrix $a_{\mathrm{du}, x_{-}}^{\circ \bigcirc}$. Property 1 guarantees that, for

$$
|a|>a^{2}+\frac{|b f|}{1-\alpha},
$$

we can make the replacement

$$
a_{\mathrm{du}, x_{-}}^{\bigcirc \bigcirc} \longmapsto p_{x_{-}, 0}^{\bigcirc}+a \sum_{k=1}^{x_{-}} p_{x_{-}, k}^{\bigcirc}
$$

with an error bounded in operator norm $\left\|r_{1, x_{-}}\right\|$by $a^{2}+|b f| /(1-\alpha)$. The replacement (71) makes the calculation of correlations extremely easy. Let us illustrate this by considering the diagram on the rhs of Eq. (32), which becomes

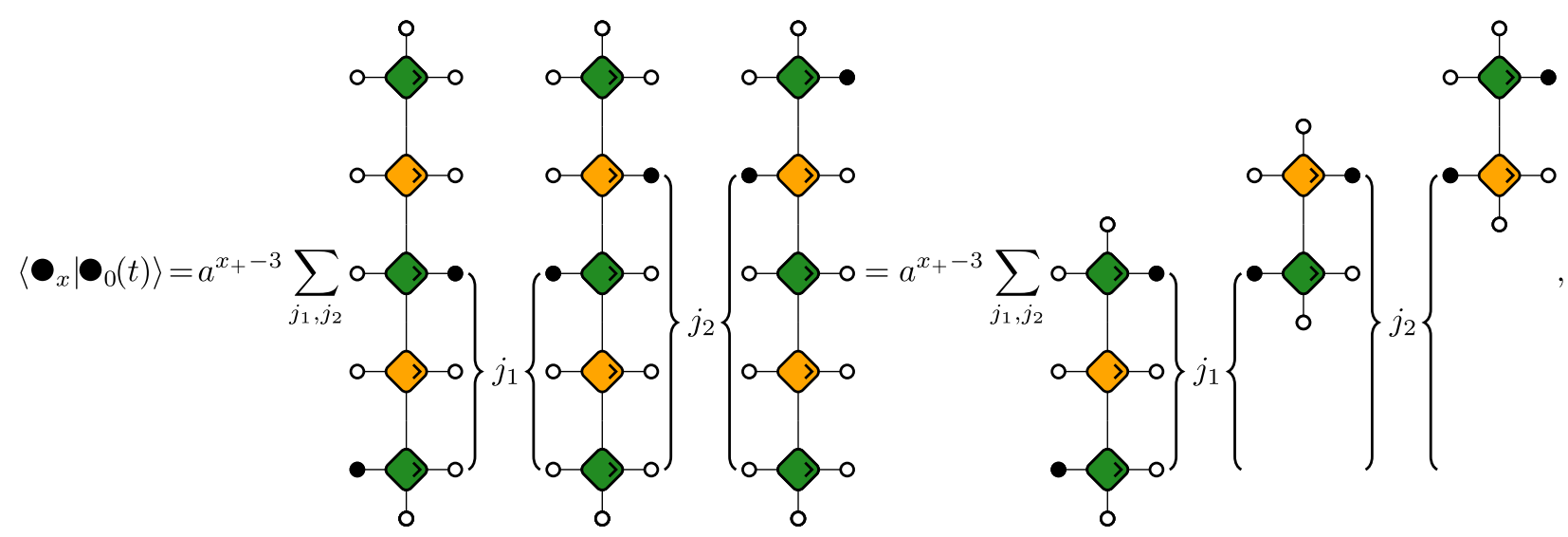

where in the second step we use the unitality relations (11) to contract the vertical lines. Using the explicit form of the one-dimensional transfer matrices

$$
a_{1}^{\circ}=\circ \bigcirc \circ=\left(\begin{array}{ll}
1 & 0 \\
0 & c
\end{array}\right), \quad a_{1}^{\bullet \circ}=\bullet \bigcirc \circ=\left(\begin{array}{cc}
0 & 0 \\
q \varepsilon & d
\end{array}\right),
$$

$$
a_{1}^{\circ \bullet}=\circ \bullet \cdot\left(\begin{array}{cc}
0 & p \varepsilon \\
0 & e
\end{array}\right), a_{1}^{\bullet \bullet}=\bullet \bullet \bullet=\left(\begin{array}{ll}
a & b \\
f & g
\end{array}\right),
$$

and of the ones depicted with orange gates that are obtained by setting $\varepsilon=0$ in the above, we see that Eq. (72) is nothing but the sum (38) of skeleton diagrams.

Taking into account the bound on the norm of $\left\|r_{1, x}\right\|$, we find that the error associated with the replacement (71) is bounded by

$$
\mathcal{O}\left(\left[a^{2}+\frac{|b f|}{1-\alpha}\right]^{\nu_{+}} a^{x_{+}-\nu_{+}}\right)
$$

so that Eq. (72) becomes exact in the limit $x_{+}, \nu_{+} \rightarrow \infty$ with $\tilde{x}_{+}$fixed. This bound is obtained by replacing just one of the vertical dual-unitary strips with the remainder $r_{1, x}$.

Specifically, considering the relative error

$$
R\left(x_{+}, x_{-}\right)=\left|\frac{\left.\left\langle\boldsymbol{\bullet}_{x} \mid \boldsymbol{\bullet}_{0}(t)\right\rangle\right|_{\mathrm{sk}}-\left\langle\boldsymbol{\bullet}_{x} \mid \boldsymbol{\bullet}_{0}(t)\right\rangle}{\left\langle\boldsymbol{\bullet}_{x} \mid \boldsymbol{\bullet}_{0}(t)\right\rangle}\right|,
$$

where $\left\langle\boldsymbol{\bigcirc}_{x} \mid \boldsymbol{\bigcirc}_{0}(t)\right\rangle$ is the exact result and $\left.\left\langle\boldsymbol{\bigcirc}_{x} \mid \boldsymbol{\bigcirc}_{0}(t)\right\rangle\right|_{\text {sk }}$ is the skeleton expression [Eq. (38)], we get

$$
R\left(x_{+}, x_{-}\right)=\mathcal{O}\left(\left[a^{2}+\frac{|b f|}{1-\alpha}\right]^{\nu_{+}} a^{-\nu_{+}}\right) \text {. }
$$

Thus, Eq. (38) gives a good approximation to the full result when $\nu_{+}$is large enough.

A completely analogous reasoning holds for horizontal strips $\nu_{-} \times x_{+}$, whenever 


$$
|c|>c^{2}+\frac{|d e|}{1-\beta},
$$

and $\nu_{-}$is large enough. In particular, for $x_{-}, \nu_{-} \rightarrow \infty$ with $\tilde{x}_{-}$fixed, we again find an exact statement.

We stress that in the above argument we never use the fact that the defects are disposed along a regular sublattice: We just use that their minimal separation $\bar{\nu}_{ \pm}$in one of the two directions becomes large. Provided that the latter condition applies, the correlations are sums of skeleton diagrams. In the case of irregular disposition of defects, however, one has to sum over only the skeleton diagrams connecting the (irregular) subset of lattice sites containing defects. Hence, the combinatorial factors differ from those in Eq. (38).

Finally, we note that all this admits a simple interpretation in terms of the paths introduced in the previous subsection. The different eigenvectors of the transfer matrix can be seen as increasingly thicker horizontal lines of length 1 . Their contributions - the eigenvalues of $a_{\mathrm{du}, x}^{\circ}$ - are obtained by complicated combinations of the tiles (57), which can be interpreted as a complicated "dressing." In this language, Property 1 implies that if Eq. (70) holds, the dominant weight is carried by the "bare" propagator

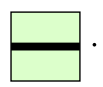

\section{Unit density, small strength}

Now let us consider a different perturbative limit: small strengths $\varepsilon \ll 1$ and fixed density. In particular, for definiteness, we focus on the case of unit density $\delta=1$, which is the most interesting from the physical point of view. Namely, we take all the gates in the diagram (32) to be green and set

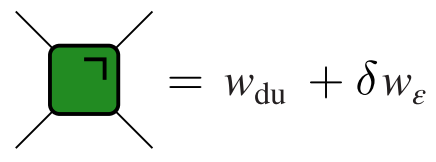

with

$$
w_{\mathrm{du}}:=\left(\begin{array}{cccc}
1 & 0 & 0 & 0 \\
0 & 0 & a & b \\
0 & c & 0 & d \\
0 & e & f & g
\end{array}\right), \quad \delta w_{\varepsilon}:=\varepsilon\left(\begin{array}{cccc}
0 & 0 & 0 & 0 \\
0 & p & 0 & 0 \\
0 & 0 & q & 0 \\
0 & 0 & 0 & 0
\end{array}\right),
$$

where we make sure that $w_{\mathrm{du}}+\delta w_{\varepsilon}$ is a bona fide $\mathrm{U}(1)$ averaged unitary gate by imposing that it becomes bistochastic when conjugated with $H \otimes H$ (cf. the remark right before Sec. IVA).

The advantage of the parametrization (80) is that in this way-in the language of Sec. IV A-parameter $\varepsilon$ "counts the turns." Namely, each fixed order in perturbation theory is determined by the sum of all allowed paths with a fixed number of turns. Even though this result is useful for classifying possible terms of different perturbative orders, the explicit evaluation of each order appears far from trivial.

Let us first consider the simplest case: the leading order that is proportional to $\varepsilon$, when $x \in \mathbb{Z}+\frac{1}{2}, y \in \mathbb{Z}$. It is given by a single skeleton diagram:

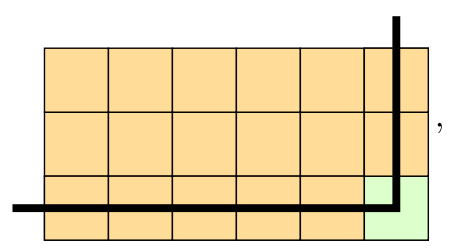

and there are no other diagrams of the same order. Therefore, the relative error for $\varepsilon \rightarrow 0$ goes as $\mathcal{O}(\varepsilon)$, even for small $x_{-}$or $x_{+}$.

In contrast, the first nontrivial order in the case $x, y \in \mathbb{Z}$ and $x_{+} \gg x_{-}>1$ is of the order of $\varepsilon^{2}$ and has much more complex structure. A simple set of contributions to this order is given by skeleton diagrams of the form

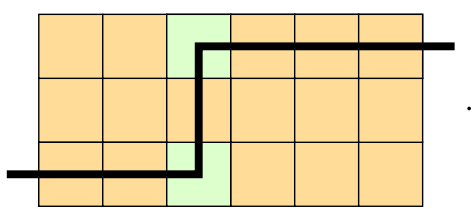

Still, many more diagrams contribute at the same order in perturbation theory. For example, we have

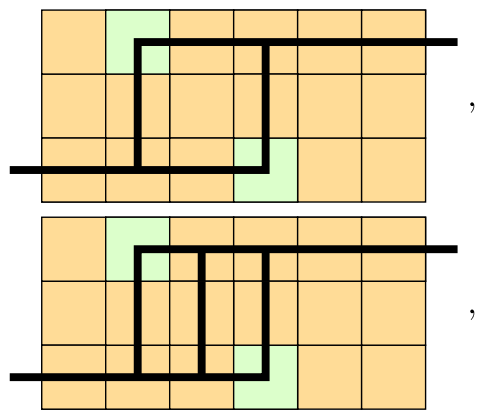

The crucial observation at this point is that there exists a regime for the parameters of the gate $w_{\mathrm{du}}$, where all these "complicated" diagrams give negligible contribution when both $x_{-}$and $x_{+}$become large. Specifically, this situation happens when both of the conditions discussed in the previous section-Eqs. (70) and (78)-hold. This observation can be understood as follows. First, we note that the 
diagrams in Eq. (84) can be thought of as skeleton diagrams with a dressed horizontal one-particle propagator. Then, we observe that, as we are working at a fixed order in perturbation theory, these dressed propagators are composed of dual-unitary tiles only. We can then make use of Property 1 to bound their contribution by

$$
\text { const }\left(a^{2}+\frac{|f d|}{1-\alpha}\right)^{x}
$$

where $x \gg 1$ is the length of the corresponding segment of the dressed propagator. We see that for large enough $x$ this contribution is exponentially suppressed with respect to the "bare" line $\left(\propto a^{x}\right)$. If both conditions (70) and (78) hold, this reasoning can be repeated at any fixed order in perturbation theory to show that the skeleton diagrams are leading when $x_{+}$and $x_{-}$are both large.

To check the above reasoning, we perform a direct numerical evaluation of the correlation function (13) and compare it with the prediction (38), obtained by summing all skeleton diagrams. The comparison is extremely encouraging: The deviations are typically undetectable on the scale of the plot; see Fig. 3 for a representative example. The technical details of numerical simulations are discussed in Appendix F.

Turning to a more quantitative analysis of the agreement, we consider the relative error (76) where $\left\langle\boldsymbol{\ominus}_{x} \mid \boldsymbol{\bigcirc}_{0}(t)\right\rangle$ is calculated numerically with no approximations while $\left.\left\langle\boldsymbol{\bigcirc}_{x} \mid \boldsymbol{\Theta}_{0}(t)\right\rangle\right|_{\text {sk }}$ is calculated using Eq. (38). The results are reported in Figs. 4-6. Specifically, Fig. 4 concerns the case of large $x_{+}=x_{-}$. We see that, when the "unperturbed gate" $w_{\mathrm{du}}$ fulfills the conditions (70) and (78), the relative error is always very small and appears to vanish with $\varepsilon$. Interestingly, even if the predictions (38) for $x \in \mathbb{Z}$ and $x \in \mathbb{Z}+1 / 2$ are of different orders in $\varepsilon$, we observe almost the same relative errors in the two cases. Lastly, an

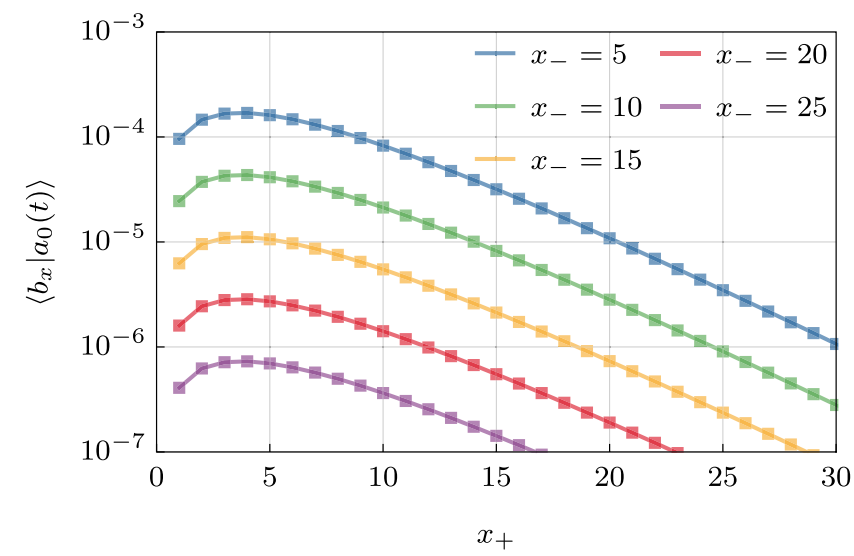

FIG. 3. Exact correlations computed numerically (solid lines) and the prediction of Eq. (38) (squares) for $x \in \mathbb{Z}$ for a quantum circuit with elementary gate given by gate 2 of Table I and maximal density of defects $\delta=1$.

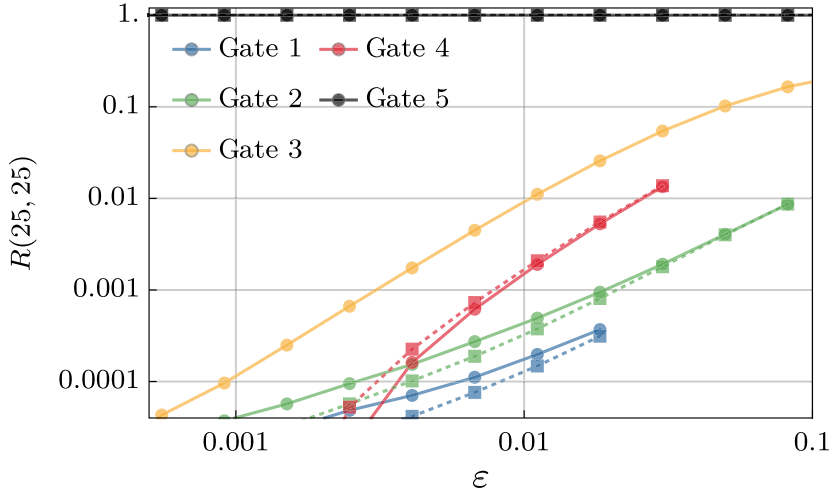

FIG. 4. Relative error $R\left(x_{+}, x_{-}\right)$[cf. Eq. (76)] for $x_{+}=x_{-}$as a function of $\varepsilon$, with maximal density of defects $\delta=1$. Full and dashed lines, respectively, correspond to integer and half-oddinteger end points $\left(x \in \mathbb{Z}, \mathbb{Z}+\frac{1}{2}\right)$, while different colors correspond to different gates (specified in Table I). Note that the gate corresponding to the black line does not fulfill the condition (70). For each gate, we stop at the value of $\varepsilon$, at which the gate ceases to be bistochastic.

important point highlighted by Fig. 4 is that the relative error is of the order of one for any $\varepsilon$ when the conditions (70) and (78) are violated.

Our argument above relies on the fact that $x_{-}$and $x_{+}$are both large. When one of the two, say, $x_{-}$, is fixed, we expect the relative error to be $\mathcal{O}\left(\varepsilon^{0}\right)$ in the case $x, y \in \mathbb{Z}$ $[\mathcal{O}(\varepsilon)$ for the case of integer and half-integer end points] and bounded by Eq. (85) with $y=x_{-}$. For small $x_{-}$, this result can be directly verified by computing the exact correlations through Eq. (18). For example, in the case of $x_{-}=2$ and $x \in \mathbb{Z}$, we find

$$
\begin{aligned}
\left\langle\boldsymbol{\bullet}_{x} \mid \boldsymbol{\bigcirc}_{0}(t)\right\rangle= & p q \varepsilon^{2}\left(\begin{array}{c}
x_{+} \\
1
\end{array}\right) a^{x_{+}-1}\left(1-\frac{b f}{a^{2}+b f-a}\right) \\
& -\frac{p q \varepsilon^{2} b f\left[a^{x_{+}}-\left(a^{2}+b f\right)^{x_{+}}\right]}{\left(a^{2}+b f-a\right)^{2}} .
\end{aligned}
$$

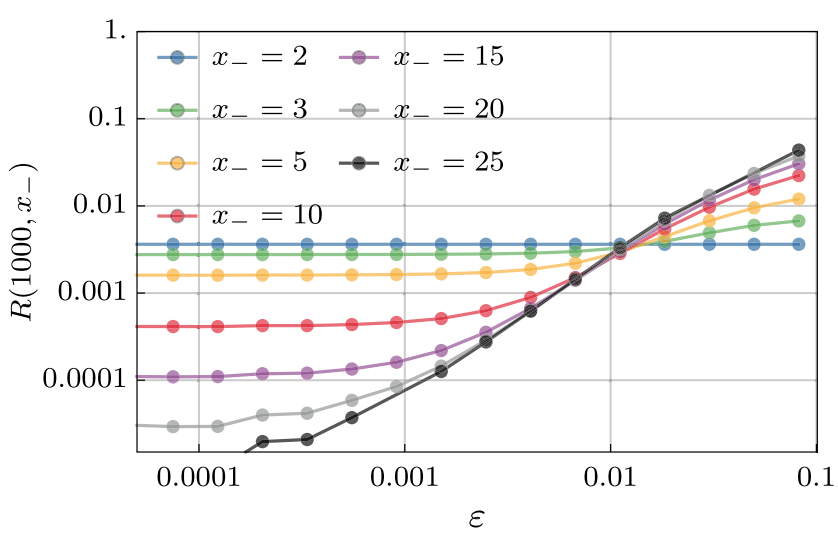

FIG. 5. Relative error $R\left(x_{+}, x_{-}\right)$[cf. Eq. (76)] for $x_{-} \ll x_{+}$as a function of $\varepsilon$. The gate is fixed to gate 2 from Table I for $x \in \mathbb{Z}$ and density of defects $\delta=1$. 


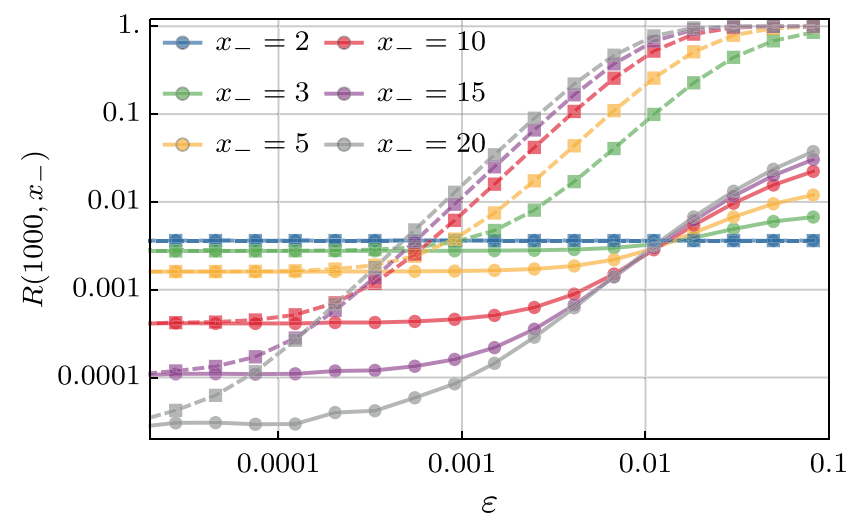

FIG. 6. Relative errors $R\left(x_{+}, x_{-}\right)$(solid curves) and $R_{1}\left(x_{+}, x_{-}\right)$ (dashed curves) versus $\varepsilon . R\left(x_{+}, x_{-}\right)$is computed using Eq. (76), while $R_{1}\left(x_{+}, x_{-}\right)$is computed by a modified version of Eq. (76) where the skeleton-diagram contribution (38) is replaced by its first nontrivial order in $\varepsilon$. The gate used for the numerical experiments is gate 2 from Table I for $x \in \mathbb{Z}$ and density of defects $\delta=1$.

This result shows that the leading correction is a dressing of the skeleton diagram in the "short direction" and gives

$$
R\left(x_{+}, 2\right) \approx \frac{|b f|}{\left(a^{2}+b f-a\right)}, \quad x_{+} \gg 1 .
$$

This point is confirmed by our numerical results, as illustrated in Fig. 5. For increasing values of $x_{-}$, the order zero contribution becomes increasingly small and is eventually dominated by the $\mathcal{O}(\varepsilon)$ contributions.

Finally, an interesting question concerns how many orders in $\varepsilon$ one has to keep in the skeleton-diagram expansion (38) in order to get an accurate result. This question is considered in Fig. 6, where we compare the relative errors obtained by (i) keeping all orders in the expansion (38) and (ii) keeping only the leading order in $\varepsilon$. We see that prediction (i) is much more accurate for larger $\varepsilon$, even though the two predictions coincide for small enough $\varepsilon$.

\section{GENERIC GATES}

In this section, we use a combination of numerical and analytical arguments to show that the main mechanisms observed in the minimal setting of the previous section carry over to the case of generic clean quantum gates. Under certain conditions on the unperturbed gate $W_{\mathrm{du}}$, the correlations computed by summing all skeleton diagrams still agree strikingly well with the exact numerical results. To simplify our numerical studies, we consider the case of qubits $(d=2)$, where the double gate $W$ is a $16 \times 16$ matrix.

We begin by briefly describing how to compute the contribution (43) of all skeleton diagrams for the case of fixed defects on regular sublattices. The rest of the gates are dual unitary. First, we construct a single skeleton diagram by drawing a zigzag line connecting the two operators on the lattice (32) and multiply the appropriate one-dimensional transfer matrices along the line [cf. Eq. (33)]. The total contribution is obtained by summing the contributions of all possible paths on the lattice connecting the two end points and containing no left and down turns. In particular, $l_{j}^{+}\left(l_{j}^{-}\right)$in Eq. (43) are of the lengths of each horizontal (vertical) segment, and the constraints (46) come from the simple requirement that the sum of all horizontal (vertical) segments is equal to $x_{+}\left(x_{-}\right)$minus the number of turns. Note that, because of the noncommutativity of the matrix product, Eq. (43) is considerably harder to evaluate than Eq. (38). The problem of its evaluation is addressed in Appendix E.

Following the analysis of the previous section, we now consider small densities of defects and investigate the spectra of horizontal and vertical transfer matrices, $A_{\mathrm{du}, x}^{\circ \bigcirc}$ and $C_{\mathrm{du}, x}^{\circ \mathrm{O}}$, composed only of dual-unitary gates $W_{\mathrm{du}, \eta}$. We remind the reader that, as discussed in Sec. II A, these transfer matrices have the spectrum contained on the closed unit disk and a "trivial" eigenvector- $|O\rangle^{\otimes x}$ —corresponding to the eigenvalue 1 . This state, however, does not affect the correlation functions. One can see this result by considering Eq. (18) and observing that $|O\rangle^{\otimes x}$ has zero overlap with the states $|a \bigcirc \ldots \bigcirc\rangle,|\bigcirc \ldots \bigcirc b\rangle, A_{x}^{\bigcirc b}|\bigcirc \ldots \bigcirc\rangle$, $C_{x}^{a}|\bigcirc \ldots \bigcirc\rangle$. The relevant quantity for correlations is then the next-to-leading eigenvalue. In analogy with the discussion in Sec. IV C 1, we conclude that if (i) the next-toleading eigenvalue $\lambda_{1}$ of $A_{\mathrm{du}, x}^{\bigcirc \bigcirc}$ has eigenvectors with support one-i.e., it is of the form

$$
|\underbrace{\bigcirc \ldots \bigcirc}_{k} a \underbrace{\bigcirc \ldots \bigcirc}_{x-k-1}\rangle, \quad k=1, \ldots, x
$$

—and (ii) there is a finite gap (in magnitude) between $\lambda_{1}$ and the rest of the spectrum, the skeleton diagrams give a good approximation to the correlation functions for large enough $\bar{\nu}_{+}$(minimal separation of the defects in the horizontal direction). Relative errors are bounded by

$$
\text { const }\left(\frac{\lambda_{2}}{\lambda_{1}}\right)^{\bar{\nu}_{+}}
$$

where $\lambda_{2}$ is the third leading eigenvalue of $A_{\mathrm{du}, x}^{\circ \mathrm{O}}$. Note that, in the language of the previous section, this result still corresponds to saying that the bare propagator carries the dominant weight. Analogous statements hold for the vertical direction, if one replaces $A_{\mathrm{du}, x}^{\bigcirc \bigcirc}$ by $C_{\mathrm{du}, x}^{\circ \bigcirc}$ and $\bar{\nu}_{+}$ by $\bar{\nu}_{-}$.

In this more complicated setting, we are not able to prove a rigorous statement, such as Property 1 , to determine the set of gates for which (i) and (ii) hold. Nevertheless, investigating the spectrum numerically, we find strong 

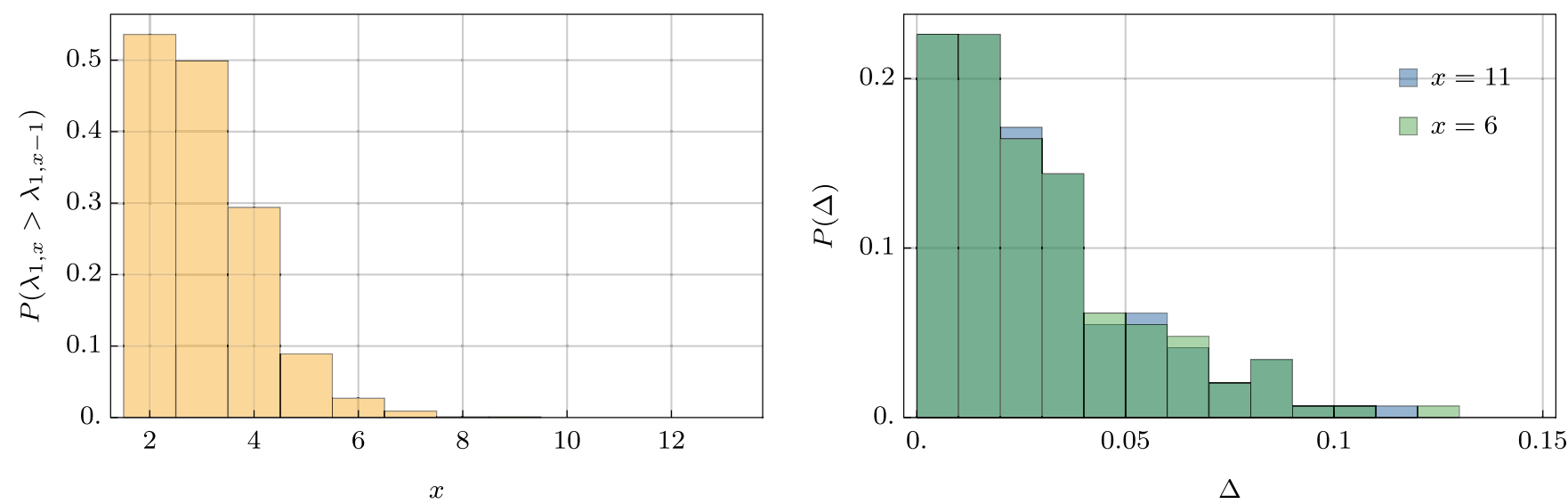

FIG. 7. Left: probability of increase of the first subleading eigenvalue of the transfer matrix $A_{\mathrm{du}, x}^{\bigcirc \bigcirc}$ when increasing the size of $A_{\mathrm{du}, x}^{\circ \bigcirc}$ from $x-1$ to $x$. The histogram is generated by considering 1000 matrices (100 for $x=12,13$ ) at fixed $J_{1,2}=\pi / 4, J_{3}=0.1$ and Haar random $\mathrm{U}(2)$ matrices $u_{i}, i=1,2,3,4$ [see the parametrization (53)] and searching for the largest eigenvalues of the transfer matrix up to $x=13$. Right: probability distribution of the transfer matrix's gap $\Delta=\lambda_{1}-\lambda_{2}$, for the 148 cases, which show no increase of the first subleading eigenvalue.

evidence that a class of gates, for which the leading nontrivial eigenvectors are of the form (88), exists; see Fig. 7 (left). Moreover, focusing on this class, we isolate a subclass that shows a finite gap: An example of the probability distribution for the gap is reported in Fig. 7 (right) for two values of $x$ (i.e., the length of the transfer matrix). Finally, we remark that Fig. 7 (left) also shows a rapid drop in the probability that a certain nontrivial eigenvector has a leading eigenvalue, with the size of its support. This result suggests that if (i) or (ii) does not hold, the dominant contribution to the correlations is still given by skeleton diagrams. In general, however, these diagrams have "thickened" lines corresponding to dressed propagators: The width of the line corresponds to the support of the leading eigenvector.

Finally, we consider maximal density $\delta=1$ and small $\eta$. In complete analogy with the discussion in Sec. IV C 2, we decompose the gate $W_{\eta}$ in two parts as follows:

$$
W_{\eta}=\overbrace{}^{\prime}=W_{\mathrm{du}, \eta}+\delta W_{\eta}
$$

where $W_{\mathrm{du}, \eta}$ is dual unitary and the only nonzero elements of $\delta W_{\eta}(18$ matrix elements in total) are the "turns"
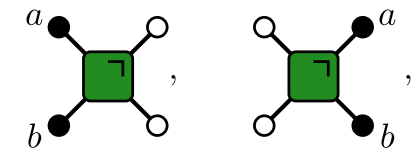

$$
a, b=\sigma^{x}, \sigma^{y}, \sigma^{z} .
$$

Note that both the elements of $W_{\mathrm{du}, \eta}$ and those of $\delta W_{\eta}$, depend on $\eta$ in general. The difference is that for small $\eta$ the former are $\mathcal{O}\left(\eta^{0}\right)$, while the latter are $\mathcal{O}(\eta)$.

Equipped with the definition (90), we are now in a position to repeat the argument of Sec. IV C 2 by formally considering a perturbative expansion in the number of turns (which at the first order is equivalent to that in $\eta$ ). We then conclude that, if points (i) and (ii) above hold for both $A_{\mathrm{du}, x}^{\bigcirc \bigcirc}$ and $C_{\mathrm{du}, x}^{\circ \circ}$, Eq. (43) gives the leading contribution at each fixed order in $\eta$ when $x_{+}$and $x_{-}$are both large. This result is in agreement with our numerical findings: Representative examples are reported in Figs. 8 and 9. Specifically, Fig. 8 (left) and Fig. 9 show excellent agreement between the prediction of Eq. (43) and the exact numerical results for two different examples.

(a) A symmetric quantum circuit with local gate

$$
U=(u \otimes u) V\left[\frac{\pi}{4}+\eta, \frac{\pi}{4}+\eta, 0.1\right](v \otimes v),
$$

where $V\left[\left\{J_{i}\right\}\right]$ is defined in Eq. (54), while $u$ and $v$ are reported in Table II. This gate can be brought to the form (30) with the dual-unitary part and perturbation explicitly given by, respectively,

$$
U_{\mathrm{du}}=(u \otimes u) V\left[\frac{\pi}{4}, \frac{\pi}{4}, 0.1\right](v \otimes v),
$$

$$
P=\left(v^{\dagger} \sigma^{x} v\right) \otimes\left(v^{\dagger} \sigma^{x} v\right)+\left(v^{\dagger} \sigma^{y} v\right) \otimes\left(v^{\dagger} \sigma^{y} v\right) .
$$

We verify numerically that $A_{\mathrm{du}, x}^{\circ \bigcirc}$ (equal to $C_{\mathrm{du}, x}^{\circ \circ}$ as the gate is symmetric) constructed with the dual-unitary part of the gate fulfills (i) and (ii).

(b) The kicked Ising chain $[64,65]$. Namely, we consider the local gate $[11,16]$

$U[J, B, h]=U_{I}[J, h] e^{-i B\left(\sigma^{r} \otimes \mathbb{1}+1 \otimes \sigma^{r}\right)} U_{I}[J, h]$,

with 

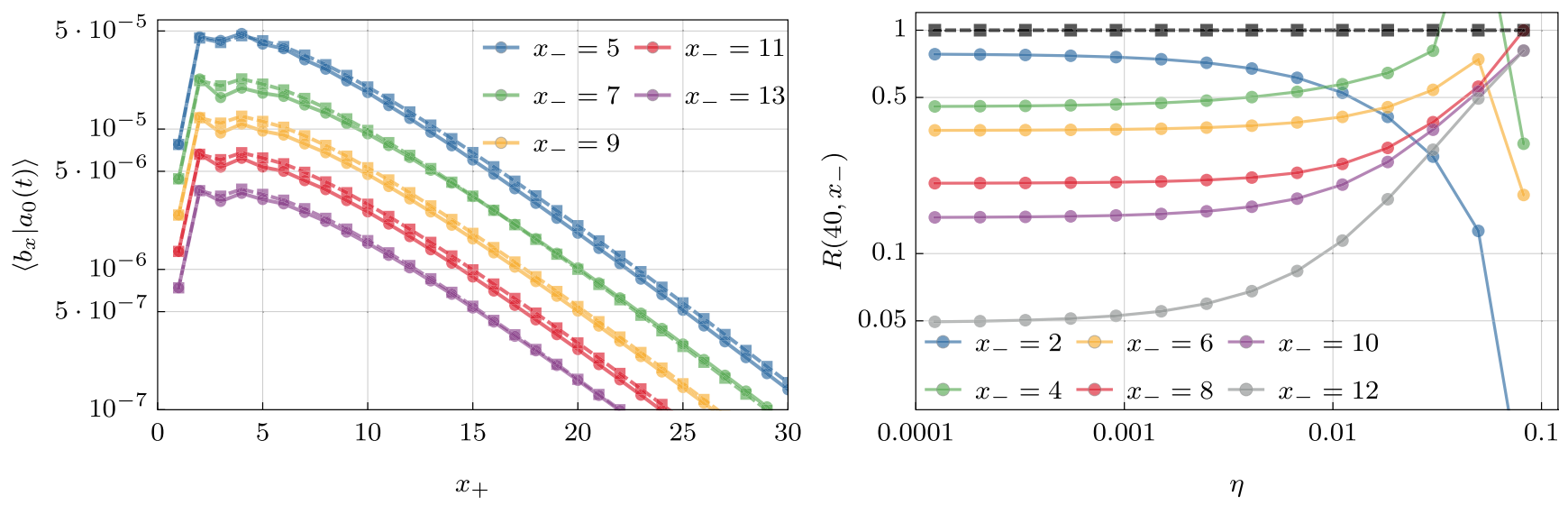

FIG. 8. Left: correlation functions of operators $a=b=\sigma^{x}$ at integer sites versus $x_{+}$at $\eta=0.011109$. Solid lines are numerical results, and dashed lines are the predictions of Eq. (43). Right: relative errors versus $\eta$ at different $x_{-}$. A gate that does not fulfill Eq. (88) is drawn in black. The results are for symmetric generic gates, as specified in Table II, with maximal density of defects $\delta=1$.

$$
U_{I}[J, h]=e^{-i J\left(\sigma^{z} \otimes \sigma^{z}\right)-i(h / 2)\left(\sigma^{z} \otimes 1+1 \otimes \sigma^{z}\right)} .
$$

The model is dual unitary for $B=J=\pi / 4$, while here we consider $B=J=\pi / 4+\eta$. In this case, the gate can again be written in the form (30) with $U_{\mathrm{du}}=$ $U[\pi / 4, \pi / 4, h]$ (we do not report the explicit form of $P$ because it is unwieldy). Interestingly, we find that, depending on parameter $h$, the transfer matrix $A_{\mathrm{du}, x}^{\circ \mathrm{O}}$ $\left(=C_{\mathrm{du}, x}^{\circ}\right)$ can either fulfill (i) or not. Moreover, the additional structure of the kicked Ising model (see Sec. 5.3 of Ref. [13]) generates additional eigenvectors associated to $\lambda_{1}$ with support strictly larger than one (although small). Therefore, even when (i) holds, (ii) is not strictly valid. However, it is reasonable to assume that the eigenvector with support 1 gives the leading contribution, as it has the largest overlap with local

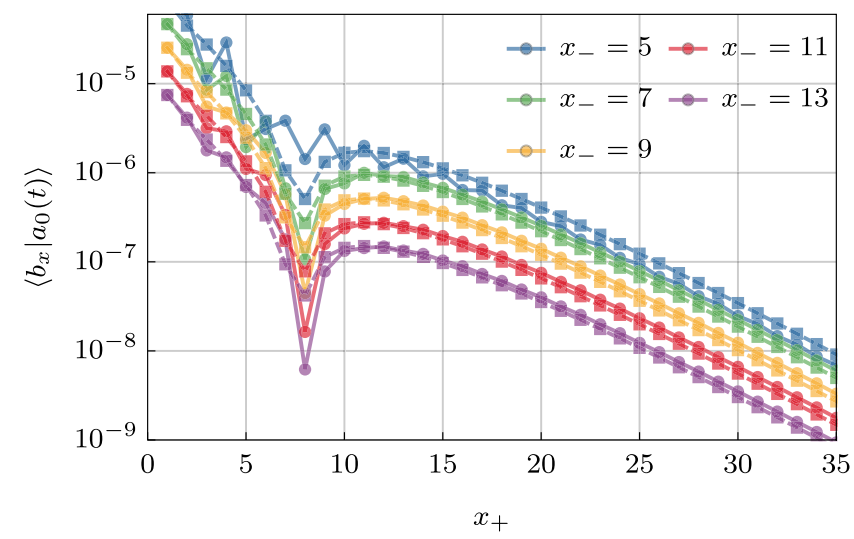

FIG. 9. Correlation functions of operators $a=b=\sigma^{x}$ at integer sites versus $x_{+}$for a kicked Ising spin chain. Solid lines are numerical results, and dashed lines are the predictions of Eq. (43). The results are for a kicked Ising spin chain at $J=B=(\pi / 4)+0.01, h=1.2$, as defined in Eq. (95) with density of defects $\delta=1$. operators. This assumption is confirmed by our numerical results (see Fig. 9): Considering a value of $h$ for which (i) holds, we find excellent agreement between the prediction Eq. (43) and exact numerics. More detailed information on the comparison between Eq. (43) and the numerical results is reported in Fig. 8 (right) and in Fig. 10. In particular, the latter shows that for fixed $\eta$ and large $x_{+}$the relative error decreases with $x_{-}$. This decrease is in agreement with our expectations [cf. Eqs. (85) and (89)] of an error bounded by

$$
\text { const }\left(\frac{\lambda_{2}}{\lambda_{1}}\right)^{x_{-}}
$$

In fact, Fig. 10 shows an even faster decay, suggesting that the bound is not tight. Finally, from the black line in Fig. 8

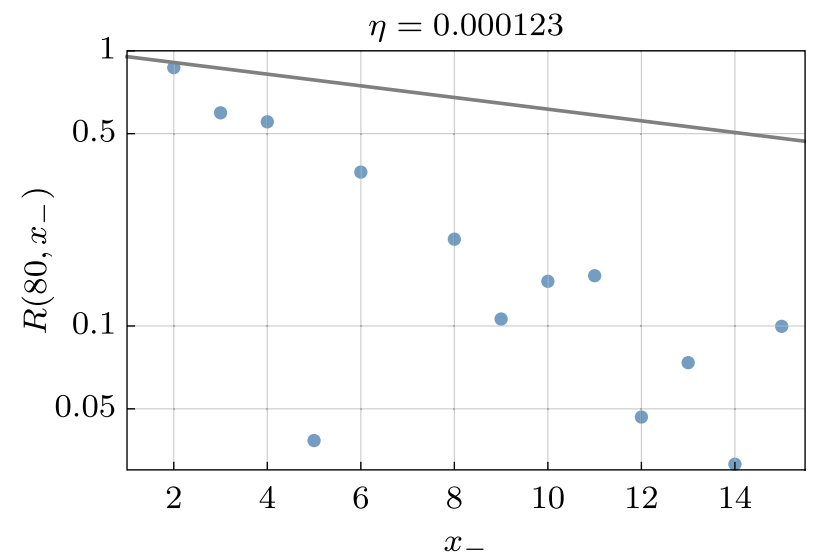

FIG. 10. Relative error for the correlations among $\sigma^{x}$ operators at integer sites versus $x_{-}$at large $x_{+}$and small $\eta$. The gray line reports the upper bound (97). The results are for symmetric generic gate fulfilling Eq. (88), as specified in Table II, with density of defects $\delta=1$. The point at $x_{-}=7$ has a relative error smaller than $0.3 \%$ and is not shown. 
(right), we see that if conditions (i) and (ii) are violated, the agreement is immediately absent.

\section{CONCLUSIONS AND PERSPECTIVES}

In this paper, we studied correlation functions in perturbed dual-unitary circuits or, equivalently, in perturbed dual-bistochastic Markov chains. We considered the problem for three increasingly more realistic (increasingly complex) settings - clean systems with random defects, noisy systems with fixed defects, and clean systems with fixed defects - and identified a class of dual-unitary circuits that is stable under perturbations. More precisely, when these systems are perturbed, their correlations continue to be given in terms of one-dimensional transfer matrices (single-qudit maps) and, hence, to (generically) decay exponentially. These exponentially decaying correlations are continuous with respect to dual-unitarity-breaking perturbations demonstrating the structural stability. The main qualitative change induced by the perturbations is that they allow the correlations to spread through the whole causal light cone and not just along its edge, as occurs in the pure dual-unitary case. One can intuitively think of the perturbations as defects that permit the correlations "to turn" in spacetime. Remarkably, the quantitative features of the correlations are extremely well captured by a simple "path-integral" formula, corresponding to the sum of correlations over all one-dimensional paths within the causal light cone that connect the end points. In conclusion, this paper presents, to our knowledge, the first set of theoretical results on the stability of the ergodic regime of quantum many-body systems under generic perturbations and opens the research arena of quantum many-body ergodic theory.

The case of noisy systems with fixed defects turned out to be an intriguing minimal model. It shows all the physical features of the generic setting but simultaneously allows for rigorous derivations. This case is not isolated (see, e.g., Ref. [15]): Introducing a small degree of randomness to isolate minimal settings appears to be a fruitful route for accessing nontrivial information about interacting many-body systems. In the minimal setting, we identified four additional classes of circuits-besides the dual-unitary ones-where correlation functions are exactly solvable-i.e., the aforementioned path-integral formula applies exactly. Systems in these classes are generically strongly interacting and generate highly complex dynamics. An obvious direction for future research is to study them further, understanding, for example, their spectral statistics and their nonequilibrium dynamics. Moreover, it would be very interesting to understand whether these classes can be defined in generic quantum circuits.

Another outstanding question raised by our work concerns what happens when one perturbs dual-unitary circuits that are not in the stable class. One possibility, which seems to be hinted by our numerical results, is that the correlation functions continue to have a path-integral form but the paths are thickened [66]. In other words, they are computed in terms of transfer matrices for a chain of width $n$ rather than of width 1 . If $n$ does not scale with time-as we seem to observe, in general- the physical picture remains very similar to the one studied here and, in particular, all correlations continue to decay exponentially. However, it would be interesting to understand whether there exists a class of circuits for which $n$ grows with time. This class could lead to a phase transition in the behavior of correlations and, therefore, to richer physics.

We can immediately propose two generalizations of our setup. First, instead of unitary quantum circuits, one can consider circuits of completely positive maps. These include the class of circuits with projective measurements that is currently attracting substantial attention, as it displays measurement-driven phase transitions [49-51,67]. Specifically, it is straightforward to generalize the concept of dual bistochasticity to dual quantum bistochasticity. Second, our treatment can be directly extended to perturbed dual-unitary (or dual-bistochastic) circuits in higher spatial dimensions where generic circuits display nontrivial complexity transitions [68]. In this case, we again expect the correlations to be written as sums over onedimensional paths.

\section{ACKNOWLEDGMENTS}

The work has been supported by Advanced grant of European Research Council (ERC) No. 694544_OMNES and the program P1-0402 of Slovenian Research Agency.

\section{APPENDIX A: DUAL-BISTOCHASTIC MARKOV CIRCUITS}

In this Appendix, we show that the object (12) can be interpreted as the correlation function of a Markov circuit, i.e., a discrete time classical Markov chain where at each half time step the time evolution couples only nearest neighbors with bistochastic matrices. To see this interpretation, let us regard Eq. (12) as the fundamental object, forgetting its origin in terms of the $\mathrm{U}(1)$ noise averaged quantum circuit and focus on the following setting.

(i) Each wire in the tensor network (12) has generic dimension $N \in \mathbb{N}$, not restricted to squares of positive integers. In particular, we choose a certain basis

$$
\{|\alpha\rangle: \alpha=1, \ldots, N\}
$$

and interpret each state as a possible state of a classical spin (or any abstract configuration of a classical system). Thus, we now view our physical system as a chain of $2 L$ classical spins. The 
probability distributions over configurations of such chain can be formally expanded in the product basis

$$
\left\{\left|\alpha_{1}, \ldots, \alpha_{2 L}\right\rangle: \alpha_{i}=1, \ldots, N\right\}
$$

Namely, we can write

$$
|p(t)\rangle=\sum_{\left\{\alpha_{j}\right\} \in\{1 \ldots N\}^{2 L}} p\left(t ;\left\{\alpha_{j}\right\}\right)\left|\alpha_{1}, \ldots, \alpha_{2 L}\right\rangle,
$$

where the coefficient $p\left(t ;\left\{\alpha_{j}\right\}\right)$ gives the probability that the system is in the configuration $\alpha_{1}, \ldots, \alpha_{2 L}$ at time $t$, and, hence,

$$
p\left(t ;\left\{\alpha_{j}\right\}\right) \in[0,1] \text { and } \sum_{\left\{\alpha_{j}\right\} \in\{1 \ldots N\}^{2 L}} p\left(t ;\left\{\alpha_{j}\right\}\right)=1
$$

(ii) The state $|O\rangle$ has the following expansion in the basis (A1):

$$
|\bigcirc\rangle:=\frac{1}{\sqrt{N}} \sum_{\alpha=1}^{N}|\alpha\rangle
$$

Namely, $|\bigcirc\rangle$ is the flat sum of all possible configurations of a single spin, and, apart from a normalization factor, it represents the flat probability distribution. In the context of classical stochastic processes, such a state is known as the maximal entropy state and is typically denoted by $|\omega\rangle$. Therefore, restoring the correct normalization, we have

$$
|\bigcirc\rangle=\sqrt{N}|\omega\rangle .
$$

(iii) The local two-body gate $W$ is not unitary but bistochastic in the tensor product of two bases (A1). Specifically,

$0 \leq\left\langle\alpha \beta|W| \alpha^{\prime} \beta^{\prime}\right\rangle \leq 1, \quad \forall \alpha, \beta, \alpha^{\prime}, \beta^{\prime}$,

$\sum_{\alpha, \beta=1}^{N}\left\langle\alpha \beta|W| \alpha^{\prime} \beta^{\prime}\right\rangle=1=\sum_{\alpha, \beta=1}^{N}\left\langle\alpha^{\prime} \beta^{\prime}|W| \alpha \beta\right\rangle$,

$\forall \alpha^{\prime}, \beta^{\prime}$.

Therefore, W in Eq. (19) is now a Markov chain propagator evolving probability distributions of the configurations of a chain of $2 L$ classical spins (or any other discrete variable degrees of freedom). More precisely, one can define

$$
|p(t+1)\rangle=\mathbb{W}|p(t)\rangle
$$

as the time-evolved probability distribution.

The bistochastic property (A7) of the elementary local gate $W$ implies that the maximal entropy state is stationary: $W|\omega\rangle \otimes|\omega\rangle=|\omega\rangle \otimes|\omega\rangle$. This result means that the unitality relations (11) are satisfied even if the gate is not unitary, and we recover the natural light-cone causal structure leading to Eq. (13) in the thermodynamic limit.

In this language, Eq. (12) is the correlation function in the maximal entropy state of two (diagonal) local observables

$$
A=\sum_{\alpha} A_{\alpha}|\alpha\rangle\left\langle\alpha\left|, \quad B=\sum_{\alpha} B_{\alpha}\right| \alpha\right\rangle\langle\alpha|,
$$

such that

$$
A|\bigcirc\rangle=|a\rangle, \quad B|\bigcirc\rangle=|b\rangle,
$$

where $|a\rangle=\sum_{\alpha} A_{\alpha}|\alpha\rangle$ and $|b\rangle=\sum_{\beta} B_{\beta}|\beta\rangle$ are the states appearing in Eq. (12). In other words, we can rewrite the rhs of Eq. (12) as

$$
\left\langle b_{x+y} \mid a_{y}(t)\right\rangle=N^{2 L}\left\langle\omega \ldots \omega\left|B_{x+y} \mathbb{W}^{t} A_{y}\right| \omega \ldots \omega\right\rangle,
$$

which is the expression for dynamical correlation functions in classical Markov chains and classical cellular automata [69-72].

Moreover, defining the "dual" local Markov gate $\tilde{W}$ by

$$
\left\langle\alpha \beta|\tilde{W}| \alpha^{\prime} \beta^{\prime}\right\rangle=\left\langle\beta^{\prime} \beta|W| \alpha^{\prime} \alpha\right\rangle
$$

and requiring it to be bistochastic in the basis $\{|\alpha \beta\rangle \equiv|\alpha\rangle \otimes|\beta\rangle\}$, the dual-unitality relations (20) are also satisfied. This result immediately implies that-if both $W$ and $\tilde{W}$ are bistochastic - the correlations take the simple form (29). Indeed, the simplification of the correlation functions is based only on the diagrammatic relations (11) and (20) without utilizing the unitarity of the gates. We refer to bistochastic gates $W_{\mathrm{du}}$ with this special property as dual bistochastic.

\section{Reduced gates as Markov circuits with $N=2$}

Hereby, we can now establish a direct connection with the U(1)-averaged unitary gates studied in Sec. IV. Consider the minimal case where each classical spin takes only $N=2$ values and define $|\mathbf{O}\rangle$ as the state orthogonal to $|O\rangle$, i.e.,

$$
|\mathbf{O}\rangle:=\frac{1}{\sqrt{2}}(|1\rangle-|2\rangle)
$$

Using the second requirement in Eq. (A7), it is immediate to verify the following.

Property 2.-Let the gate $w$ be bistochastic in the basis $\left\{\left|\alpha_{i}\right\rangle \otimes\left|\alpha_{j}\right\rangle\right\}_{i, j=1}^{2}$, and then 
(a) $w$ takes the form (35) when expressed in the basis $\{|\bigcirc \bigcirc\rangle,|\bigcirc \bigcirc\rangle,|\bigcirc\rangle\rangle,|\bigcirc\rangle\rangle\}$. The explicit parametrization of the matrix elements is reported in Appen$\operatorname{dix} \mathrm{B} 2$.

(b) If $w$ is dual bistochastic, it takes the form (36) in the basis $\{|\bigcirc \bigcirc\rangle,|\bigcirc \bigcirc,| \bigcirc\rangle\rangle,|\bigcirc\rangle\}$. The explicit parametrization of the matrix elements is again reported in Appendix B 2.

(c) The matrix implementing the change of basis from $\{|\bigcirc \bigcirc\rangle,|\bigcirc \bigcirc\rangle,|\bigcirc \bigcirc\rangle,|\bigcirc\rangle\rangle\}$ to $\left\{\left|\alpha_{i}\right\rangle \otimes\left|\alpha_{j}\right\rangle\right\}$ is given by $H \otimes H$, where $H$ is the Hadamard transformation [cf. Eq. (37)].

\section{APPENDIX B: PARAMETRIZATION OF THE GATES (35) AND (36)}

In this Appendix, we present an explicit parametrization of the gates (35) and (36) depending on whether they are obtained as U(1)-averaged (dual-)unitary double gates or as conjugated (dual-)bistochastic ones.

\section{U(1)-averaged (dual-)unitary double gates}

To find a convenient parametrization, we note that the elements of the gate (35) can be computed by evaluating

$$
\left\langle o_{1}, o_{2}|w| o_{3}, o_{4}\right\rangle=\operatorname{tr}\left[\left(o_{1} \otimes o_{2}\right) \bar{U}^{\dagger}\left(o_{3} \otimes o_{4}\right) \bar{U}\right], \quad o_{1}, o_{2}, o_{3}, o_{4}=\mathbb{1}, \sigma^{z}
$$

Here, we define

$$
\bar{U}=\left(u\left(0, \beta_{1}, \gamma_{1}\right) \otimes u\left(0, \beta_{2}, \gamma_{2}\right)\right) V\left[\left\{J_{i}\right\}\right]\left(u\left(0, \beta_{3}, \gamma_{3}\right)^{\dagger} \otimes u\left(0, \beta_{4}, \gamma_{4}\right)^{\dagger}\right),
$$

where $u(\alpha, \beta, \gamma)$ is defined in Eq. (55) and $V\left[\left\{J_{i}\right\}\right]$ in Eq. (54). Equation (B1) follows directly from the parametrization (53) and the form (51) of the averaged gate.

In particular, explicit calculations yield

$$
\begin{aligned}
& p \varepsilon=\cos \left(2 J_{1}\right) \cos \left(2 J_{2}\right) C_{3}^{(2)} C_{3}^{(4)}+\cos \left(2 J_{1}\right) \cos \left(2 J_{3}\right) C_{2}^{(2)} C_{2}^{(4)}+\cos \left(2 J_{2}\right) \cos \left(2 J_{3}\right) C_{1}^{(2)} C_{1}^{(4)}, \\
& q \varepsilon=\cos \left(2 J_{1}\right) \cos \left(2 J_{2}\right) C_{3}^{(1)} C_{3}^{(3)}+\cos \left(2 J_{1}\right) \cos \left(2 J_{3}\right) C_{2}^{(1)} C_{2}^{(3)}+\cos \left(2 J_{2}\right) \cos \left(2 J_{3}\right) C_{1}^{(1)} C_{1}^{(3)}, \\
& a=\sin \left(2 J_{1}\right) \sin \left(2 J_{2}\right) C_{3}^{(2)} C_{3}^{(3)}+\sin \left(2 J_{1}\right) \sin \left(2 J_{3}\right) C_{2}^{(2)} C_{2}^{(3)}+\sin \left(2 J_{2}\right) \sin \left(2 J_{3}\right) C_{1}^{(2)} C_{1}^{(3)}, \\
& c=\sin \left(2 J_{1}\right) \sin \left(2 J_{2}\right) C_{3}^{(1)} C_{3}^{(4)}+\sin \left(2 J_{1}\right) \sin \left(2 J_{3}\right) C_{2}^{(1)} C_{2}^{(4)}+\sin \left(2 J_{2}\right) \sin \left(2 J_{3}\right) C_{1}^{(1)} C_{1}^{(4)}, \\
& b=\sum_{\alpha, \beta, \gamma=1}^{3} \sin \left(2 J_{\beta}\right) \cos \left(2 J_{\gamma}\right) \mathcal{E}^{\alpha \beta \gamma} C_{\alpha}^{(2)} C_{\beta}^{(3)} C_{\gamma}^{(4)}, \quad d=-\sum_{\alpha, \beta, \gamma=1}^{3} \cos \left(2 J_{\beta}\right) \sin \left(2 J_{\gamma}\right) \mathcal{E}^{\alpha \beta \gamma} C_{\alpha}^{(1)} C_{\beta}^{(3)} C_{\gamma}^{(4)}, \\
& e=-\sum_{\alpha, \beta, \gamma=1}^{3} \sin \left(2 J_{\alpha}\right) \cos \left(2 J_{\beta}\right) \mathcal{E}^{\alpha \beta \gamma} C_{\alpha}^{(1)} C_{\beta}^{(2)} C_{\gamma}^{(4)}, \quad f=\sum_{\alpha, \beta, \gamma=1}^{3} \cos \left(2 J_{\alpha}\right) \sin \left(2 J_{\beta}\right) \mathcal{E}^{\alpha \beta \gamma} C_{\alpha}^{(1)} C_{\beta}^{(2)} C_{\gamma}^{(3)} \text {, } \\
& g=\sum_{\beta=1}^{3} C_{\beta}^{(1)} C_{\beta}^{(2)} C_{\beta}^{(3)} C_{\beta}^{(4)}+\cos \left(2 J_{1}\right) \cos \left(2 J_{2}\right) \sum_{\alpha \neq \beta=1}^{2} C_{\beta}^{(1)} C_{\alpha}^{(2)} C_{\beta}^{(3)} C_{\alpha}^{(4)}+\sin \left(2 J_{1}\right) \sin \left(2 J_{2}\right) \sum_{\alpha \neq \beta=1}^{2} C_{\beta}^{(1)} C_{\alpha}^{(2)} C_{\alpha}^{(3)} C_{\beta}^{(4)} \\
& +\cos \left(2 J_{1}\right) \cos \left(2 J_{3}\right) \sum_{\alpha \neq \beta=1,3} C_{\beta}^{(1)} C_{\alpha}^{(2)} C_{\beta}^{(3)} C_{\alpha}^{(4)}+\sin \left(2 J_{1}\right) \sin \left(2 J_{3}\right) \sum_{\alpha \neq \beta=1,3} C_{\beta}^{(1)} C_{\alpha}^{(2)} C_{\alpha}^{(3)} C_{\beta}^{(4)} \\
& +\cos \left(2 J_{2}\right) \cos \left(2 J_{3}\right) \sum_{\alpha \neq \beta=2,3} C_{\beta}^{(1)} C_{\alpha}^{(2)} C_{\beta}^{(3)} C_{\alpha}^{(4)}+\sin \left(2 J_{2}\right) \sin \left(2 J_{3}\right) \sum_{\alpha \neq \beta=2,3} C_{\beta}^{(1)} C_{\alpha}^{(2)} C_{\alpha}^{(3)} C_{\beta}^{(4)} \text {, }
\end{aligned}
$$

where $\mathcal{E}^{\alpha \beta \gamma}$ is the three-dimensional Levi-Civita symbol and we define

$$
\begin{array}{cc}
C_{1}^{(i)}=\sin \left(\beta_{i}\right) \cos \left(\gamma_{i}\right), & C_{2}^{(i)}=\sin \left(\beta_{i}\right) \sin \left(\gamma_{i}\right), \quad C_{3}^{(i)}=\cos \left(\beta_{i}\right), \quad i=1,2, \\
C_{1}^{(i)}=-\sin \left(\beta_{i}\right) \cos \left(\gamma_{i}\right), \quad C_{2}^{(i)}=\sin \left(\beta_{i}\right) \sin \left(\gamma_{i}\right), \quad C_{3}^{(i)}=\cos \left(\beta_{i}\right), \quad i=3,4 .
\end{array}
$$


The parametrization of Eq. (36) follows by replacing $J_{1}$ and $J_{2}$ by $\pi / 4$. Explicitly, we have

$$
\begin{gathered}
p \varepsilon=0=q \varepsilon, \\
a=C_{3}^{(2)} C_{3}^{(3)}+\sin \left(2 J_{3}\right)\left(C_{2}^{(2)} C_{2}^{(3)}+C_{1}^{(2)} C_{1}^{(3)}\right), \quad c=C_{3}^{(1)} C_{3}^{(4)}+\sin \left(2 J_{3}\right)\left(C_{2}^{(1)} C_{2}^{(4)}+C_{1}^{(1)} C_{1}^{(4)}\right), \\
b=\cos \left(2 J_{3}\right) C_{3}^{(4)}\left(C_{1}^{(2)} C_{2}^{(3)}-C_{2}^{(2)} C_{1}^{(3)}\right), \quad d=\cos \left(2 J_{3}\right) C_{3}^{(3)}\left(C_{1}^{(1)} C_{2}^{(4)}-C_{2}^{(1)} C_{1}^{(4)}\right), \\
e=\cos \left(2 J_{3}\right) C_{3}^{(2)}\left(C_{1}^{(1)} C_{2}^{(4)}-C_{2}^{(1)} C_{1}^{(4)}\right), \quad f=\cos \left(2 J_{3}\right) C_{3}^{(1)}\left(C_{1}^{(2)} C_{2}^{(3)}-C_{2}^{(2)} C_{1}^{(3)}\right), \\
g=\sum_{\beta=1}^{3} C_{\beta}^{(1)} C_{\beta}^{(2)} C_{\beta}^{(3)} C_{\beta}^{(4)}+\sum_{\alpha \neq \beta=1}^{2} C_{\beta}^{(1)} C_{\alpha}^{(2)} C_{\alpha}^{(3)} C_{\beta}^{(4)}+\sin \left(2 J_{3}\right)\left[\sum_{\alpha \neq \beta=1,3} C_{\beta}^{(1)} C_{\alpha}^{(2)} C_{\alpha}^{(3)} C_{\beta}^{(4)}+\sum_{\alpha \neq \beta=2,3} C_{\beta}^{(1)} C_{\alpha}^{(2)} C_{\alpha}^{(3)} C_{\beta}^{(4)}\right] .
\end{gathered}
$$

Noting that

$$
\begin{aligned}
& \left(u\left(0, \beta_{1}, \gamma_{1}\right) \otimes u\left(0, \beta_{2}, \gamma_{2}\right)\right) V\left[\left\{\pi / 4, \pi / 4, J_{3}\right\}\right]\left(u\left(0, \beta_{3}, \gamma_{3}\right)^{\dagger} \otimes u\left(0, \beta_{4}, \gamma_{4}\right)^{\dagger}\right) \\
& \quad=\left(u\left(0, \beta_{1}, 0\right) \otimes u\left(0, \beta_{2}, 0\right)\right) V\left[\left\{\pi / 4, \pi / 4, J_{3}\right\}\right]\left(u\left(0, \beta_{3}, \gamma_{3}-\gamma_{2}\right)^{\dagger} \otimes u\left(0, \beta_{4}, \gamma_{4}-\gamma_{1}\right)^{\dagger}\right)
\end{aligned}
$$

we can set

$$
\gamma_{1}=\gamma_{2}=0
$$

and (B12)-(B16) further simplify to

$$
p \varepsilon=0=q \varepsilon
$$

$a=\cos \left(\beta_{2}\right) \cos \left(\beta_{3}\right)-\sin \left(2 J_{3}\right) \sin \left(\beta_{2}\right) \sin \left(\beta_{3}\right) \cos \left(\gamma_{3}\right), \quad c=\cos \left(\beta_{1}\right) \cos \left(\beta_{1}\right)-\sin \left(2 J_{3}\right) \sin \left(\beta_{1}\right) \sin \left(\beta_{4}\right) \cos \left(\gamma_{4}\right)$,

$$
\begin{aligned}
b= & \cos \left(2 J_{3}\right) \cos \left(\beta_{4}\right) \sin \left(\beta_{2}\right) \sin \left(\beta_{3}\right) \sin \left(\gamma_{3}\right), \quad d=\cos \left(2 J_{3}\right) \cos \left(\beta_{3}\right) \sin \left(\beta_{1}\right) \sin \left(\beta_{4}\right) \sin \left(\gamma_{4}\right), \\
e & =\cos \left(2 J_{3}\right) \cos \left(\beta_{2}\right) \sin \left(\beta_{1}\right) \sin \left(\beta_{4}\right) \sin \left(\gamma_{4}\right), \quad f=\cos \left(2 J_{3}\right) \cos \left(\beta_{1}\right) \sin \left(\beta_{2}\right) \sin \left(\beta_{3}\right) \sin \left(\gamma_{3}\right) \\
g= & \cos \left(\beta_{1}\right) \cos \left(\beta_{2}\right) \cos \left(\beta_{3}\right) \cos \left(\beta_{4}\right)+\sin \left(\beta_{1}\right) \sin \left(\beta_{2}\right) \sin \left(\beta_{3}\right) \sin \left(\beta_{4}\right) \cos \left(\gamma_{3}\right) \cos \left(\gamma_{4}\right) \\
& -\sin \left(2 J_{3}\right)\left[\sin \left(\beta_{1}\right) \cos \left(\beta_{2}\right) \cos \left(\beta_{3}\right) \sin \left(\beta_{4}\right) \cos \left(\gamma_{4}\right)+\cos \left(\beta_{1}\right) \sin \left(\beta_{2}\right) \sin \left(\beta_{3}\right) \cos \left(\beta_{4}\right) \cos \left(\gamma_{3}\right)\right]
\end{aligned}
$$

\section{Conjugated (dual-)bistochastic gates}

Considering a $4 \times 4$ bistochastic matrix

$$
B=\left(\begin{array}{cccc}
x_{11} & x_{12} & x_{13} & 1-\sum_{j} x_{1 j} \\
x_{21} & x_{22} & x_{23} & 1-\sum_{j} x_{2 j} \\
x_{31} & x_{32} & x_{33} & 1-\sum_{j} x_{3 j} \\
1-\sum_{i} x_{i 1} & 1-\sum_{i} x_{i 2} & 1-\sum_{i} x_{i 3} & \sum_{i j} x_{i j}-2
\end{array}\right)
$$

parametrized by elements of a convex set

$$
\mathcal{R}_{b}=\left\{\left(x_{11}, x_{12}, \ldots, x_{33}\right) ; \quad x_{i j} \in[0,1], \quad\left(0 \leq \sum_{i} x_{i j} \leq 1\right) \wedge\left(0 \leq \sum_{i} x_{j i} \leq 1\right), \quad j=1,2,3\right\}
$$


Conjugating Eq. (B24) with $H \otimes H$, we obtain a gate of the form (35) with coefficients

$$
\begin{array}{crr}
p \varepsilon=x_{11}+x_{13}+x_{31}+x_{33}-1, & a=x_{11}+x_{12}+x_{31}+x_{32}-1, & b=1-x_{12}-x_{13}-x_{32}-x_{33}, \\
c=x_{11}+x_{13}+x_{21}+x_{23}-1, & q \varepsilon=x_{11}+x_{12}+x_{21}+x_{22}-1, & d=1-x_{12}-x_{13}-x_{22}-x_{23}, \\
e=1-x_{21}-x_{23}-x_{31}-x_{33}, & f=1-x_{21}-x_{22}-x_{31}-x_{32}, & g=x_{22}+x_{23}+x_{32}+x_{33}-1 .
\end{array}
$$

Instead, a dual-bistochastic matrix is written as

$$
B_{\mathrm{db}}=\left(\begin{array}{cccc}
x_{11} & x_{12} & x_{13} & 1-\sum_{j} x_{1 j} \\
x_{21} & 1-x_{21}-x_{12}-x_{11} & x_{23} & x_{11}+x_{12}-x_{23} \\
x_{31} & x_{32} & 1-x_{31}-x_{13}-x_{11} & x_{11}+x_{13}-x_{32} \\
1-\sum_{i} x_{i 1} & x_{11}+x_{21}-x_{32} & x_{11}+x_{31}-x_{23} & x_{23}+x_{32}-x_{11}
\end{array}\right),
$$

the set of which is parametrized by a seven-dimensional convex set

$\mathcal{R}_{\mathrm{db}}=\left\{\left(x_{11}, x_{12}, x_{21}, x_{13}, x_{31}, x_{23}, x_{32}\right) ;\left(x_{11}, x_{12}, x_{13}, x_{21}, 1-x_{12}-x_{21}-x_{11}, x_{23}, x_{31}, x_{32}, 1-x_{13}-x_{32}-x_{11} \in \mathcal{R}_{b}\right)\right\}$.

In this case, the parametrization (B26)-(B28) simplifies to

$$
\begin{aligned}
p \varepsilon=0, \quad a=x_{11}+x_{12}+x_{31}+x_{32}-1, & b=x_{31}+x_{11}-x_{12}-x_{32}, \\
c=x_{11}+x_{13}+x_{21}+x_{23}-1, \quad q \varepsilon=0, \quad d=x_{21}+x_{11}-x_{13}-x_{23}, & \\
e=x_{13}+x_{11}-x_{21}-x_{23}, \quad f=x_{12}+x_{11}-x_{31}-x_{32}, & g=1-\sum_{k}\left(x_{k 1}+x_{1 k}\right)+x_{23}+x_{32} .
\end{aligned}
$$

\section{APPENDIX C: GUE-AVERAGED GATE}

In this Appendix, we treat analytically the problem outlined in Sec. III considering correlations averaged over random defects. Specifically, we take $P$ in Eq. (30) to be random matrices independently distributed at each spacetime point according to the Gaussian unitary ensemble, i.e.,

$$
P_{\mathrm{GUE}}(P)=\mathcal{N}_{d} e^{-\left(d^{2} / 2\right) \operatorname{tr}\left[(P-\mu 1)(P-\mu 1)^{\dagger}\right]}
$$

where $\mathcal{N}_{d}$ is a normalization constant, $d^{2}$ is the dimension of $P$, and $\mu$ is the disorder mean, while the variance of matrix elements $\sigma^{2}$ is fixed to unity. This result is not a restriction, as the "strength" of the disorder is already controlled by the parameter $\eta$ and all physical quantities depend on $\eta$ and $\sigma$ only through $\eta / \sigma$.

We start by proving the following property.

Property 3.-The average of the doubled gate $W_{\eta}$ reads as

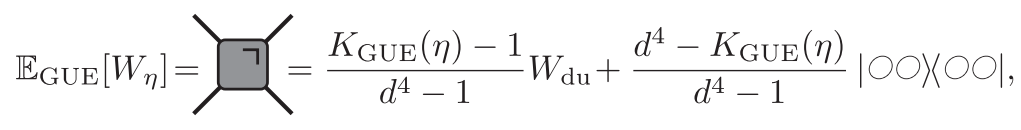

where $E_{\mathrm{GUE}}[\cdot]$ is the average over Eq. $(\mathrm{C} 1)$ and $K_{\mathrm{GUE}}(x) \equiv \mathbb{E}_{\mathrm{GUE}}\left[\left|\operatorname{tr}\left[e^{i x P}\right]\right|^{2}\right]$ is the spectral form factor of the Gaussian unitary ensemble. 
Proof.-Since we have

$$
\mathbb{E}_{\mathrm{GUE}}\left[W_{\eta}\right]=\mathbb{E}_{\mathrm{GUE}}\left[\left(e^{-i \eta P} \otimes e^{i \eta P^{T}}\right)\right] W_{\mathrm{du}},
$$

we can focus on the first term in the product:

$$
M \equiv \mathbb{E}_{\mathrm{GUE}}\left[\left(e^{-i \eta P} \otimes e^{i \eta P^{T}}\right)\right] .
$$

We now observe that $M$ is invariant under conjugation with $U \otimes U^{*}$, where $U$ is a $d^{2} \times d^{2}$ unitary matrix and $(\cdot)^{*}$ represents complex conjugation in the canonical basis (1). The Schur's lemma implies

$$
M=\alpha P_{\text {sym }}+\beta P_{\text {antisym }},
$$

where $P_{\text {sym }}$ and $P_{\text {antisym }}$ are the projectors on the spaces of the irreps in which the tensor product of two conjugated fundamental representations of $U\left(d^{2}\right)$ decomposes. Specifically, these two representations are $d^{4}-1$ - and one-dimensional, and in our notations we have

$P_{\text {sym }}=\mathbb{1}-|\bigcirc \bigcirc\rangle\left\langle\bigcirc \bigcirc\left|, \quad P_{\text {antisym }}=\right| \bigcirc \bigcirc\right\rangle\langle\bigcirc \bigcirc|$.

The coefficients are readily computed by multiplying $M$ by $P_{\text {sym }}$ and $P_{\text {antisym }}$, respectively, and taking the trace. This result leads to

$$
\alpha=\frac{\operatorname{tr}[M]-1}{\operatorname{tr}\left[P_{\mathrm{sym}}\right]}=\frac{K_{\mathrm{GUE}}(\eta)-1}{d^{4}-1}, \quad \beta=1 .
$$

Plugging back into Eq. (C3) and using $W_{\mathrm{du}} P_{\text {antisym }}=$ $P_{\text {antisym }}$, we obtain Eq. (C2).

Since both terms on the rhs of Eq. (C2) fulfill the "dualunitality" conditions (20) and their coefficients sum to one, the averaged gate fulfills Eq. (20) as well. Then the correlations immediately reduce to the form (29), where $\left\langle a\left|\left(A_{\mathrm{du}, 1}^{\bigcirc \bigcirc}\right)^{2 t}\right| b\right\rangle$ and $\left\langle b\left|\left(C_{\mathrm{du}, 1}^{\bigcirc \bigcirc}\right)^{2 t}\right| a\right\rangle \quad$ are, respectively, replaced by

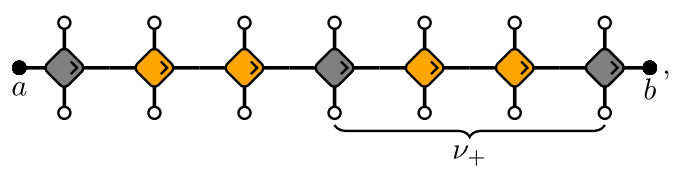

and

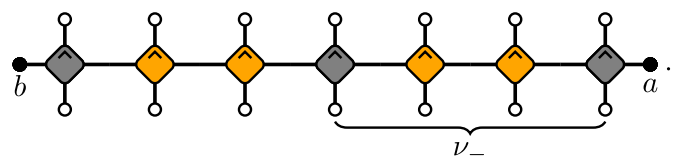

Here, we distribute the defects along the regular sublattice of Eq. (32) for a more convenient representation. The treatment of more general (e.g., random) dispositions of defects is completely analogous.

Expanding each averaged gate as in Eq. (C2), we produce a sum of $2^{\tilde{x}_{ \pm}}$terms [cf. Eq. (39)]. As can be directly verified using Eq. (11), only the term with no $|\bigcirc \bigcirc\rangle\langle\bigcirc|$ gives a nontrivial contribution. Considering that term, we directly find

$$
\begin{aligned}
\mathbb{E}_{\mathrm{GUE}}\left[\left\langle b_{x+y} \mid a_{y}(t)\right\rangle\right]= & \bmod (2 y, 2) \delta_{x+t}\left\langle b\left|\left(C_{\mathrm{du}, 1}^{\circ \bigcirc}\right)^{2 t}\right| a\right\rangle\left[\frac{K_{\mathrm{GUE}}(\eta)-1}{d^{4}-1}\right]^{\tilde{x}_{-}} \\
& +\bmod (2 y+1,2) \delta_{x-t}\left\langle a\left|\left(A_{\mathrm{du}, 1}^{\bigcirc \bigcirc}\right)^{2 t}\right| b\right\rangle\left[\frac{K_{\mathrm{GUE}}(\eta)-1}{d^{4}-1}\right]^{\tilde{x}_{+}}
\end{aligned}
$$

where $K_{\mathrm{GUE}}(t)$ can explicitly be written as [73]

$$
K_{\mathrm{GUE}}(t)=-\int d x d y e^{i t y} \rho_{2, d^{2}}^{c}(x+y, x)+\left|\int d x e^{i t x} \rho_{1, d^{2}}^{c}(x)\right|^{2}+d^{2}
$$

where

$$
\rho_{1, n}^{c}(x)=K_{n}(x, x), \quad \rho_{2, n}^{c}(x, y)=\left[K_{n}(x, y)\right]^{2}, \quad K_{n}(x, y)=\frac{e^{-\left(x^{2}+y^{2}\right) / 2}}{\pi^{1 / 2} 2^{n}(n-1) !} \frac{H_{n}(x) H_{n-1}(y)-H_{n}(y) H_{n-1}(x)}{x-y},
$$

and $H_{n}(x)$ are the Hermite polynomials. In particular, for $d=2$ we find

$$
K_{\mathrm{GUE}}(t)=4+e^{-t^{2} / 2}\left[12-24 t^{2}-\frac{23}{2} t^{4}+\frac{8}{3} t^{6}-\frac{25}{96} t^{8}+\frac{1}{96} t^{10}\right] .
$$

We conclude by observing that the GUE average considered here is very different from the flat average over the Haar measure on $U\left(d^{2}\right)$. Taking a Haar random perturbation $R$ in Eq. (30) instead of $e^{i \eta P}$ immediately gives 


$$
\mathbb{E}_{U\left(d^{2}\right)}\left[W_{\eta}\right]=\mathbb{E}_{U\left(d^{2}\right)}\left[R^{\dagger} \otimes R^{T}\right]=|\bigcirc \bigcirc\rangle\langle\bigcirc \bigcirc,
$$

where in the second step we use the right multiplication invariance of the Haar measure and the last step is a trivial instance of the known result on integrals over unitary groups [74] [it can also be obtained from Eq. (C2) by replacing $K_{\mathrm{GUE}}(\eta)$ with $K_{\mathrm{GUE}}(1)=1$ ]. A direct consequence of this result is that all correlations are trivialized by the Haar average for any density $\delta$ of defects. Interestingly, Eq. (C2) does not reproduce Eq. (C14) for $\eta \rightarrow \infty$ since

$$
\lim _{\eta \rightarrow \infty} K_{\mathrm{GUE}}(\eta)=d^{2} .
$$

This result can be justified by noting that, even though Eq. (C1) becomes flat in this limit, it gives flat weights to different variables compared to the Haar measure (roughly speaking, $P$ rather than $e^{i P}$ ) and a flat weight for one does not imply a flat weight for the other. As expected, Eqs. (C10) and (C14) agree in the limit of infinite dimensional matrices $d \rightarrow \infty$.

\section{APPENDIX D: PROOF OF PROPERTY 1}

In this Appendix, we prove Property 1. We consider the horizontal transfer matrix $a_{0, x}^{\bigcirc \bigcirc}$, as the proof for the vertical one, $c_{\mathrm{du}, x} \mathrm{O}$, is totally analogous. Let us begin by introducing the transfer matrix

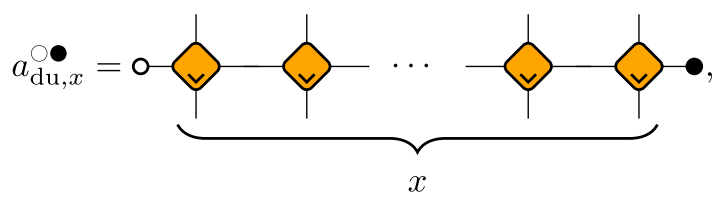

(D1)

and noting that $\left\{a_{\mathrm{du}, x}^{\circ}, a_{\mathrm{du}, x}^{\circ \bullet}\right\}$ fulfill the following recursive relations:

$$
\begin{aligned}
a_{\mathrm{du}, x}^{\circ \bigcirc}= & a_{\mathrm{du}, x-1}^{\circ \circ} \otimes\left(\begin{array}{ll}
1 & 0 \\
0 & 0
\end{array}\right)+a_{\mathrm{du}, x-1}^{\circ \circ} \otimes\left(\begin{array}{ll}
0 & 0 \\
0 & a
\end{array}\right) \\
& +a_{\mathrm{du}, x-1}^{\circ \bullet} \otimes\left(\begin{array}{ll}
0 & 0 \\
0 & b
\end{array}\right), \\
a_{\mathrm{du}, x} \bullet & a_{\mathrm{du}, x-1}^{\circ \bullet} \otimes\left(\begin{array}{ll}
c & e \\
d & g
\end{array}\right)+a_{\mathrm{du}, x-1}^{\circ \bigcirc} \otimes\left(\begin{array}{ll}
0 & 0 \\
0 & f
\end{array}\right),
\end{aligned}
$$

with

$$
a_{\mathrm{du}, 1}^{\circ \bigcirc}=\left(\begin{array}{ll}
1 & 0 \\
0 & a
\end{array}\right), \quad a_{\mathrm{du}, 1}^{\circ \bullet}=\left(\begin{array}{ll}
0 & 0 \\
0 & f
\end{array}\right) .
$$

These relations are directly established by plugging a resolution of the identity $\mathbb{1}=|O\rangle\langle O|+| \mathbf{O}\rangle\langle\mathbf{O}|$ in the rightmost connecting wire of Eqs. (62) and (D1).

Using unitality (11) and dual unitality (20), it is straightforward to prove that $|\bigcirc \ldots \bigcirc\rangle$ is an eigenvector of $a_{\mathrm{du}, x} \bigcirc$ with the eigenvalue 1 and

$$
\{|\underbrace{\bigcirc \ldots \bigcirc \bigcirc}_{k} \bigcirc \ldots\rangle\}_{k=1}^{x}
$$

are $x$ eigenvectors of $a_{\mathrm{du}, x}^{\circ \bigcirc}$ corresponding to the eigenvalue $a$. This result immediately proves the block structure of Eq. (64). Therefore, to conclude the proof, we just need to bound the norm of

$$
r_{1, x}=a_{\mathrm{du}, x}^{\circ \bigcirc}-p_{x, 0}^{\circ}-a \sum_{k=1}^{x} p_{x, k}^{\bigcirc},
$$

where the projectors $\left\{p_{x, k}^{\bigcirc}\right\}_{k=0}^{x}$ are defined in Eq. (66). We begin by considering

$$
\lambda_{1, x}:=\left\|r_{1, x}-p_{x, 0}^{\bigcirc}\right\|,
$$

where $\|A\|$ denotes the operator norm of matrix $A$. Using Eq. (D2) leads to

$$
\begin{aligned}
\lambda_{1, x} & =\max \left(\lambda_{1, x-1},\left\|a a_{\mathrm{du}, x-1}^{\circ}+b a_{\mathrm{du}, x-1}^{\odot \bullet}\right\|\right) \\
& =\max \left(\lambda_{1, x-1},|a|,\left\|a a_{\mathrm{du}, x-1}^{\circ \bigcirc}-a p_{x-1,0}^{\circ}+b a_{\mathrm{du}, x-1}^{\circ \bullet}\right\|\right),
\end{aligned}
$$

where in the second step we use that $|\bigcirc \ldots \bigcirc\rangle$ is an eigenvector of $a_{\mathrm{du}, x-1}^{\mathrm{\bullet}}$ (with eigenvalue 0). Using the triangle inequality for the operator norm on the rhs, we obtain

$$
\left\|a a_{\mathrm{du}, x-1}^{\circ \bigcirc}-a p_{x-1,0}^{\circ}+b a_{\mathrm{du}, x-1}^{\odot \bullet}\right\| \leq|a| \lambda_{1, x-1}+|b| \lambda_{x-1}^{\bullet},
$$

where we define

$$
\lambda_{x-1}^{\bullet}:=\left\|a_{\mathrm{du}, x}^{\ominus \bullet}\right\|
$$

Thus, applying triangle inequality to Eq. (D3), we find

$$
\lambda_{x}^{\bullet} \leq \alpha \lambda_{x-1}^{\bullet}+|f|,
$$

where we use that $\left\|a_{\mathrm{du}, x} \mathrm{O}\right\|=1$ (this result can be proven by the same reasoning as in Appendix A of Ref. [13]). Furthermore, we denote by $\alpha$ the operator norm of the 2 by 2 matrix in the first term on the rhs of Eq. (D3). Explicitly, $\alpha$ can be computed by considering the largest singular value of the matrix, which reads

$$
\alpha=\sqrt{\frac{c^{2}+e^{2}+g^{2}+d^{2}+\sqrt{\left(c^{2}+e^{2}-g^{2}-d^{2}\right)^{2}+4(c d+e g)^{2}}}{2}} .
$$


By means of the parameterizations (B19)-(B23) and (B31)-(B33), one can explicitly verify that $\alpha \in[0,1]$.

Using Eq. (D11), we see that $\lambda_{x}^{*}$ is bounded by $y_{x}$, which is defined as the solution of

$$
y_{x}=\alpha y_{x-1}+|f|, \quad y_{1}=|f|,
$$

and reads explicitly

$$
y_{x}=\frac{|f|}{1-\alpha}\left(1-\alpha^{x}\right) .
$$

Hence,

$$
\begin{gathered}
\left\|a a_{\mathrm{du}, x-1}^{\circ}-a P_{x-1,0}^{\bigcirc}+b a_{\mathrm{du}, x-1}^{\circ \bullet}\right\| \leq|a| \lambda_{x-1} \\
+\frac{|b f|}{1-\alpha}\left(1-\alpha^{x-1}\right) \leq|a| \lambda_{x-1}+\frac{|b f|}{1-\alpha},
\end{gathered}
$$

where in the last step we assume $\alpha<1$. Combining everything, we get

$$
\lambda_{1, x} \leq \max \left(\lambda_{1, x-1},|a|,|a| \lambda_{1, x-1}+\frac{|b f|}{1-\alpha}\right) .
$$

Considering now the range of parameters for which

$$
a^{2}+\frac{|b f|}{1-\alpha}<|a|,
$$

we have that, for $\lambda_{1, x-1} \leq|a|$, it follows $\lambda_{1, x} \leq|a|$. In particular, since $\lambda_{1,1}=|a|$, we conclude

$$
\lambda_{1, x} \leq|a| \quad \forall x .
$$

Moreover, from Eq. (D15) we conclude that

$$
\left\|a a_{\mathrm{du}, x-1}^{\bigcirc \bigcirc}-a p_{x-1,0}^{\bigcirc}+b a_{\mathrm{du}, x-1}^{\circ \bullet}\right\| \leq a^{2}+\frac{|b f|}{1-\alpha},
$$

which combined with Eq. (D8) implies

$$
\lambda_{1, x}=|a| \quad \forall x .
$$

Now we define

$$
\lambda_{2, x}:=\left\|r_{1, x}\right\|,
$$

and using Eq. (D2) we find

$$
\lambda_{2, x}=\max \left(\lambda_{2, x-1},\left\|a a_{\mathrm{du}, x-1}^{\bigcirc \bigcirc}-a p_{x-1,0}^{\bigcirc}+b a_{\mathrm{du}, x-1}^{\bigcirc \bullet}\right\|\right) .
$$

At this point, we use the bound (D19), which combined with $\lambda_{2,1}=0$ gives

$$
\lambda_{2, x} \leq a^{2}+\frac{|b f|}{1-\alpha} .
$$

This concludes the proof of Property 1.

Note that the bound (D16) is useful even when the condition (D17) is violated. Indeed, assuming $|a|<\lambda_{x}<1$, we have

$$
\lambda_{x+1} \leq \max \left(\lambda_{x},|a| \lambda_{x}+\frac{|b f|}{1-\alpha}\right) .
$$

Since $|a|<1$, this equation implies $\lambda_{x}<m^{*}$ for all $x$ and some finite $m^{*}$. Whenever $m^{*}<1$, this result produces a useful bound for the first nontrivial eigenvalue of $a_{\mathrm{du}, x}$.

\section{APPENDIX E: EVALUATION OF EQ. (43)}

To evaluate Eq. (43), one first needs to construct all lists

$$
\left\{l_{1}^{+}, \ldots, l_{n+1}^{+}\right\} ; \quad l_{j}^{+} \geq 0,
$$

and

$$
\left\{l_{1}^{-}, \ldots, l_{n}^{-}\right\} ; \quad l_{j}^{-} \geq 0,
$$

that satisfy the constraint (46). This construction is done with a recursive algorithm. At the step $k$, we generate all lists

$$
\left\{p_{1}, \ldots p_{k}\right\} ; \quad p_{j} \geq 0
$$

that satisfy

$$
\sum_{j=1}^{k} p_{j}=n_{k} \text {. }
$$

If we are generating a list with a single element $(k=1)$, this element is set by the constraint $p_{1}=n_{1}$. Otherwise, we generate lists by choosing all possible values for the last element in $p_{k} \in\left[0, \ldots, n_{k}\right]$ and solving the problem of finding all lists $\left\{p_{1}, \ldots, p_{k-1}\right\}$ with the constraint 


$$
\sum_{j=1}^{k-1} p_{j}=n_{k}-p_{k}=n_{k-1} .
$$

After all the lists are generated, we can evaluate Eq. (43).

For large $x_{ \pm}$and $n$, the above procedure becomes difficult to implement. For instance, consider the $n=n_{0}$ in the first sum of Eq. (43): This term involves the summation of

$$
\left(\begin{array}{l}
x_{+} \\
n_{0}
\end{array}\right)\left(\begin{array}{l}
x_{-}-2 \\
n_{0}-1
\end{array}\right) \approx\left(\frac{x_{+} x_{-}}{n_{0}}\right)^{n_{0}}
$$

contributions, which becomes eventually impractical for $x_{ \pm}, n_{0} \gg 1$. This problem can be overcome by (i) truncating the expansion (43) (this truncation leads to accurate results for small enough $\eta$ ) or (ii) by approximating the higher orders by substituting $A_{0,1}^{\bigcirc \mathrm{O}}$ and $C_{0,1}^{\mathrm{O}}$ with the projectors on their largest nontrivial eigenvalues, which we, respectively, denote by $\lambda_{1, a}$ and $\lambda_{1, c}$. Thus, we can rewrite the contribution of the higher orders in Eq. (43) exactly in the form Eq. (38), with the sums running from a certain $n=n_{c}\left(n=n_{c}-1\right)$ instead of $1(0)$. The parameters are expressed as

$$
\begin{aligned}
p \varepsilon & =\left\langle\lambda_{1, c}\left|\mathcal{E}_{1}\right| \lambda_{1, a}\right\rangle, \quad q \varepsilon=\left\langle\lambda_{1, a}\left|\mathcal{E}_{2}\right| \lambda_{1, c}\right\rangle, \\
a & =\lambda_{1, a}, \quad c=\lambda_{1, c},
\end{aligned}
$$

and there is an additional factor of $\left\langle b \mid \lambda_{1, a}\right\rangle\left\langle\lambda_{1, a} \mid a\right\rangle$ $\left(\left\langle b \mid \lambda_{1, c}\right\rangle\left\langle\lambda_{1, a} \mid a\right\rangle\right)$ in front of it. [In the latter expression, $|a\rangle$ and $\langle b|$ refer to initial and final operators, respectively, whereas $\left|\lambda_{1, a}\right\rangle$ and $\left|\lambda_{1, c}\right\rangle$ refer to the leading eigenvectors ( $a$ and $c$ refer to the corresponding parameters of the reduced gate).] If all $l_{j}^{ \pm} \gg 1$, this replacement gives a negligible error; however, in the sum there are also terms with $l_{j}^{ \pm} \approx 0$ and using the projector is strictly speaking unjustified. Nevertheless, this replacement gives a useful approximation, especially in the limit $x_{ \pm} \rightarrow \infty$.

\section{APPENDIX F: NUMERICAL METHODS AND PARAMETERS OF THE GATES}

Direct (brute force) numerical evaluations are performed by exactly contracting the $x_{+} \times x_{-}$tensor network (13), with the help of some basic functionalities of ITensor Library [75]. We represent the green gates as ITensor objects with the correct indices. To contract Eq. (13), we start on the right side and contract the initial state by gates in the last column one by one (first and last gates in the column are also contracted with the $\bigcirc$ state). Then, we continue in the same fashion with the remaining columns and end up with contracting with the final state.

The leading part of the spectrum of the dual-unitary transfer matrices is found using the power method, i.e., by iteration of the dual transfer matrix on a random initial vector, which is chosen to be (bi)orthogonal to the previously computed leading (left and right) eigenvectors. We circumvent the need to apply the adjoint dual-unitary transfer matrix by noticing that, for the leading eigenvectors of nontrivial support up to one, left and right eigenvectors coincide.

The parameters of the gates used in numerical experi-

\begin{tabular}{|c|c|c|c|c|c|c|c|}
\hline & $a$ & $b$ & $c$ & $d$ & $e$ & $f$ & $g$ \\
\hline Gate 1 & 0.820188 & -0.0136728 & 0.820188 & -0.0136728 & -0.0136728 & -0.0136728 & 0.679158 \\
\hline Gate 2 & 0.761132 & -0.025732 & 0.761132 & -0.025732 & -0.025732 & -0.025732 & 0.701678 \\
\hline Gate 3 & 0.0945626 & 0.0212368 & 0.195892 & 0.00603479 & 0.0000783504 & 0.271196 & 0.443805 \\
\hline Gate 4 & -0.714396 & -0.0143272 & -0.174808 & 0.139193 & -0.206479 & 0.0273134 & 0.0485487 \\
\hline Gate 5 & 0.0409352 & 0.275939 & 0.0409352 & 0.275939 & 0.275939 & 0.275939 & 0.0373261 \\
\hline
\end{tabular}
ments are given in Tables I and II.

TABLE I. Parameters of the reduced gates, which are used in the figures. In the cases where $\varepsilon$ is not stated, we use $\varepsilon=-0.002660$, -0.014746 , and 0.001 for gates 1,2 , and 3 , respectively. We use $p=q=1$ [cf. Eq. (81)].

TABLE II. Parameters of the nonaveraged gates, which are used in the figures. The gates are parametrized as $(u \otimes u) V\left[\left\{J_{i}\right\}\right](v \otimes v)$, with $V\left[\left\{J_{i}\right\}\right]$ defined in Eq. (54). The parameters are set to $J_{1}=J_{2}=\eta+\pi / 4$ and $J_{3}=0.1$.

\begin{tabular}{lccc}
\hline $\begin{array}{l}\text { Eigenvectors of } \\
\lambda_{1} \text { fulfill Eq. (88) }\end{array}$ & \multicolumn{2}{c}{$u$} & $v$ \\
\hline Yes & $\left(\begin{array}{ccc}-0.229466-0.562418 i & 0.507409-0.611202 i \\
0.316533+0.728586 i & 0.377401-0.475959 i\end{array}\right)$ & $\left(\begin{array}{cc}-0.500163-0.421942 i & -0.0342946-0.755398 i \\
0.532173-0.537209 i & -0.653978-0.0226033 i\end{array}\right)$ \\
No & $\left(\begin{array}{ccc}-0.484203+0.476337 i & 0.207991+0.70384 i \\
-0.194545+0.707674 i & -0.493189-0.467026 i\end{array}\right)$ & $\left(\begin{array}{cc}0.488082+0.160083 i & 0.0340135+0.857317 i \\
0.197049+0.835058 i & 0.427305-0.285063 i\end{array}\right)$ \\
\hline \hline
\end{tabular}


[1] A. Altland and B. Simons, Condensed Matter Field Theory (Cambridge University Press, Cambridge, England, 2010).

[2] G. D. Mahan, Condensed Matter in a Nutshell (Princeton University Press, Princeton, NJ, 2011).

[3] A. J. Daley, C. Kollath, U. Schollwoeck, and G. Vidal, Time-Dependent Density-Matrix Renormalization-Group Using Adaptive Effective Hilbert Spaces, J. Stat. Mech. (2004) P04005.

[4] D. M. Kennes and C. Karrasch, Extending the Range of Real Time Density Matrix Renormalization Group Simulations, Comput. Phys. Commun. 200, 37 (2016).

[5] T. Rakovszky, C. W. von Keyserlingk, and F. Pollmann, Dissipation-Assisted Operator Evolution Method for Capturing Hydrodynamic Transport, arXiv:2004.05177.

[6] P. Calabrese, F. H. L. Essler, and G. Mussardo, Quantum Integrability in Out of Equilibrium Systems, J. Stat. Mech. (2016) 064001.

[7] B. Bertini, F. Heidrich-Meisner, C. Karrasch, T. Prosen, R. Steinigeweg, and M. Žnidarič, Finite-Temperature Transport in One-Dimensional Quantum Lattice Models, arXiv:2003 .03334.

[8] B. Doyon, Lecture Notes on Generalised Hydrodynamics, SciPost Phys. Lect. Notes 18 (2020).

[9] F. Bonetto, J. L. Lebowitz, and L. Rey-Bellet, Fourier's Law: A Challenge for Theorists, Math. Phys. Appl. Math. 2000, 128 (2000).

[10] A. Dhar, Heat Transport in Low-Dimensional Systems, Adv. Phys. 57, 457 (2008).

[11] B. Bertini, P. Kos, and T. Prosen, Exact Correlation Functions for Dual-Unitary Lattice Models in $1+1$ Dimensions, Phys. Rev. Lett. 123, 210601 (2019).

[12] B. Bertini, P. Kos, and T. Prosen, Exact Spectral Form Factor in a Minimal Model of Many-Body Quantum Chaos, Phys. Rev. Lett. 121, 264101 (2018).

[13] B. Bertini, P. Kos, and T. Prosen, Operator Entanglement in Local Quantum Circuits I: Chaotic Dual-Unitary Circuits, SciPost Phys. 8, 067 (2020).

[14] B. Bertini, P. Kos, and T. Prosen, Operator Entanglement in Local Quantum Circuits II: Solitons in Chains of Qubits, SciPost Phys. 8, 068 (2020).

[15] B. Bertini and L. Piroli, Scrambling in Random Unitary Circuits: Exact Results, Phys. Rev. B 102, 064305 (2020).

[16] S. Gopalakrishnan and A. Lamacraft, Unitary Circuits of Finite Depth and Infinite Width from Quantum Channels, Phys. Rev. B 100, 064309 (2019).

[17] L. Piroli, B. Bertini, J. I. Cirac, and T. Prosen, Exact Dynamics in Dual-Unitary Quantum Circuits, Phys. Rev. B 101, 094304 (2020).

[18] P. W. Claeys and A. Lamacraft, Maximum Velocity Quantum Circuits, Phys. Rev. Research 2, 033032 (2020).

[19] B. Bertini, P. Kos, and T. Prosen, Entanglement Spreading in a Minimal Model of Maximal Many-Body Quantum Chaos, Phys. Rev. X 9, 021033 (2019).

[20] S. A. Rather, S. Aravinda, and A. Lakshminarayan, Creating Ensembles of Dual Unitary and Maximally Entangling Quantum Evolutions, Phys. Rev. Lett. 125, 070501 (2020).

[21] B. Gutkin, P. Braun, M. Akila, D. Waltner, and T. Guhr, Local Correlations in Dual-Unitary Kicked Chains, arXiv:2001.01298.
[22] A. Nahum, S. Vijay, and J. Haah, Operator Spreading in Random Unitary Circuits, Phys. Rev. X 8, 021014 (2018).

[23] C. W. von Keyserlingk, T. Rakovszky, F. Pollmann, and S. L. Sondhi, Operator Hydrodynamics, OTOCs, and Entanglement Growth in Systems without Conservation Laws, Phys. Rev. X 8, 021013 (2018).

[24] A. Nahum, J. Ruhman, S. Vijay, and J. Haah, Quantum Entanglement Growth under Random Unitary Dynamics, Phys. Rev. X 7, 031016 (2017).

[25] I. P. Cornfeld, S. V. Fomin, and Y. G. Sinai, Ergodic Theory (Springer, New York, 2012).

[26] V. I. Arnold and A. Avez, Ergodic Problems of Classical Mechanics, reprint edition (Addison-Wesley, New York, 1989).

[27] E. Ott, Chaos in Dynamical Systems, 2nd edition (Cambridge University Press, Cambridge, England, 2012).

[28] V. I. Arnold, Geometrical Methods in the Theory of Ordinary Differential Equations (Springer, New York, 1988).

[29] M. C. Bañuls, M. B. Hastings, F. Verstraete, and J. I. Cirac, Matrix Product States for Dynamical Simulation of Infinite Chains, Phys. Rev. Lett. 102, 240603 (2009).

[30] A. Müller-Hermes, J. I. Cirac, and M. C. Bañuls, Tensor Network Techniques for the Computation of Dynamical Observables in One-Dimensional Quantum Spin Systems, New J. Phys. 14, 075003 (2012).

[31] C. Jonay, D. A. Huse, and A. Nahum, Coarse-Grained Dynamics of Operator and State Entanglement, arXiv: 1803.00089.

[32] T. Zhou and A. Nahum, Emergent Statistical Mechanics of Entanglement in Random Unitary Circuits, Phys. Rev. B 99, 174205 (2019).

[33] T. Zhou and A. Nahum, The entanglement Membrane in Chaotic Many-Body Systems, Phys. Rev. X 10, 031066 (2020).

[34] Note that in cases (i) and (ii) only the first term on the rhs contributes in the first line. Moreover, the second line is trivial in case (ii): To find a nontrivial integer-half-oddinteger correlation, one has to look at half-odd-integer starting points in that case.

[35] P. Kos, B. Bertini, and T. Prosen, Chaos and Ergodicity in Extended Quantum Systems with Noisy Driving, arXiv: 2010.12494.

[36] T. Prosen and M. Žnidarič, Is the Efficiency of Classical Simulations of Quantum Dynamics Related to Integrability?, Phys. Rev. E 75, 015202(R) (2007).

[37] T. Prosen and I. Pižorn, Operator Space Entanglement Entropy in a Transverse Ising Chain, Phys. Rev. A 76, 032316 (2007).

[38] I. Pižorn and T. Prosen, Operator Space Entanglement Entropy in XY Spin Chains, Phys. Rev. B 79, 184416 (2009).

[39] R. Pal and A. Lakshminarayan, Entangling Power of TimeEvolution Operators in Integrable and Nonintegrable Many-Body Systems, Phys. Rev. B 98, 174304 (2018).

[40] V. Alba, J. Dubail, and M. Medenjak, Operator Entanglement in Interacting Integrable Quantum Systems: The Case of the Rule 54 Chain, Phys. Rev. Lett. 122, 250603 (2019).

[41] P. Zanardi, Entanglement of Quantum Evolutions, Phys. Rev. A 63, 040304(R) (2001). 
[42] T. Rakovszky, F. Pollmann, and C. W. von Keyserlingk, Diffusive Hydrodynamics of Out-of-Time-Ordered Correlators with Charge Conservation, Phys. Rev. X 8, 031058 (2018).

[43] V. Khemani, A. Vishwanath, and D. A. Huse, Operator Spreading and the Emergence of Dissipative Hydrodynamics under Unitary Evolution with Conservation Laws, Phys. Rev. X 8, 031057 (2018).

[44] A. Chan, A. De Luca, and J. T. Chalker, Solution of a Minimal Model for Many-Body Quantum Chaos, Phys. Rev. X 8, 041019 (2018).

[45] A. Chan, A. De Luca, and J. T. Chalker, Spectral Statistics in Spatially Extended Chaotic Quantum Many-Body Systems, Phys. Rev. Lett. 121, 060601 (2018).

[46] C. Sünderhauf, D. Pérez-García, D. A. Huse, N. Schuch, and J. I. Cirac, Localization with Random TimePeriodic Quantum Circuits, Phys. Rev. B 98, 134204 (2018).

[47] A. J. Friedman, A. Chan, A. De Luca, and J. T. Chalker, Spectral Statistics and Many-Body Quantum Chaos with Conserved Charge, Phys. Rev. Lett. 123, 210603 (2019).

[48] Y. Li, X. Chen, and M. P. A. Fisher, Measurement-Driven Entanglement Transition in Hybrid Quantum Circuits, Phys. Rev. B 100, 134306 (2019).

[49] B. Skinner, J. Ruhman, and A. Nahum, MeasurementInduced Phase Transitions in the Dynamics of Entanglement, Phys. Rev. X 9, 031009 (2019).

[50] R. Vasseur, A. C. Potter, Y.-Z. You, and A. W. W. Ludwig, Entanglement Transitions from Holographic Random Tensor Networks, Phys. Rev. B 100, 134203 (2019).

[51] A. Zabalo, M. J. Gullans, J. H. Wilson, S. Gopalakrishnan, D. A. Huse, and J.H. Pixley, Critical Properties of the Measurement-Induced Transition in Random Quantum Circuits, Phys. Rev. B 101, 060301(R) (2020).

[52] Y. Bao, S. Choi, and E. Altman, Theory of the Phase Transition in Random Unitary Circuits with Measurements, Phys. Rev. B 101, 104301 (2020).

[53] C.-M. Jian, Y.-Z. You, R. Vasseur, and A. W. W. Ludwig, Measurement-Induced Criticality in Random Quantum Circuits, Phys. Rev. B 101, 104302 (2020).

[54] Y. Li, X. Chen, and M. P. A. Fisher, Quantum Zeno Effect and the Many-Body Entanglement Transition, Phys. Rev. B 98, 205136 (2018).

[55] S. Choi, Y. Bao, X.-L. Qi, and E. Altman, Quantum Error Correction in Scrambling Dynamics and Measurement Induced Phase Transition, Phys. Rev. Lett. 125, 030505 (2020).

[56] M. J. Gullans and D. A. Huse, Dynamical Purification Phase Transition Induced by Quantum Measurements, Phys. Rev. X 10, 041020 (2020).
[57] A. Lavasani, Y. Alavirad, and M. Barkeshli, MeasurementInduced Topological Entanglement Transitions in Symmetric Random Quantum Circuits, arXiv:2004.07243.

[58] T. Rakovszky, F. Pollmann, and C. W. von Keyserlingk, Sub-ballistic Growth of Rényi Entropies due to Diffusion, Phys. Rev. Lett. 122, 250602 (2019).

[59] Y. Huang, Dynamics of Rényi Entanglement Entropy in Diffusive Qudit Systems, IOP SciNotes 1, 035205 (2020).

[60] T. Rakovszky, C. W. von Keyserlingk, and F. Pollmann, Entanglement Growth after Inhomogenous Quenches, Phys. Rev. B 100, 125139 (2019).

[61] N. Hunter-Jones, Unitary Designs from Statistical Mechanics in Random Quantum Circuits, arXiv:1905.12053.

[62] B. Kraus and J. I. Cirac, Optimal Creation of Entanglement Using a Two-Qubit Gate, Phys. Rev. A 63, 062309 (2001).

[63] F. Vatan and C. Williams, Optimal Quantum Circuits for General Two-Qubit Gates, Phys. Rev. A 69, 032315 (2004).

[64] T. Prosen, General Relation between Quantum Ergodicity and Fidelity of Quantum Dynamics, Phys. Rev. E 65, 036208 (2002).

[65] T. Prosen, Ruelle Resonances in Quantum Many-Body Dynamics, J. Phys. A 35, L737 (2002).

[66] After the first version of our paper came out, we learned of similar observations by A. Nahum, S. Roy, S. Vijay, and T. Zhou in their work (to be published): Chaos and the TimeOrdered Two-Point Function.

[67] Y. Li, X. Chen, A. W. W. Ludwig, and M. P. A. Fisher, Conformal Invariance and Quantum Non-locality in Hybrid Quantum Circuits, arXiv:2003.12721.

[68] J. Napp, R. L. La Placa, A. M. Dalzell, F. G. S. L. Brandao, and A. W. Harrow, Efficient Classical Simulation of Random Shallow 2D Quantum Circuits, arXiv:2001.00021.

[69] T. Prosen and C. Mejía-Monasterio, Integrability of a Deterministic Cellular Automaton Driven by Stochastic Boundaries, J. Phys. A 49, 185003 (2016).

[70] T. Prosen and B. Buča, Exact Matrix Product Decay Modes of a Boundary Driven Cellular Automaton, J. Phys. A 50, 395002 (2017).

[71] K. Klobas, M. Medenjak, T. Prosen, and M. Vanicat, TimeDependent Matrix Product Ansatz for Interacting Reversible Dynamics, Commun. Math. Phys. 371, 651 (2019).

[72] K. Klobas and T. Prosen, Space-like Dynamics in a Reversible Cellular Automaton, SciPost Phys. Core 2, 010 (2020).

[73] M. L. Mehta, Random Matrices and the Statistical Theory of Spectra, 2nd ed. (Academic, New York, 1991).

[74] B. Collins, Moments and Cumulants of Polynomial Random Variables on Unitary Groups, the Itzykson-Zuber Integral, and Free Probability, Int. Math. Res. Not. 2003, 953 (2003).

[75] ITensor Library (version 3), http://itensor.org. 